\title{
The dynamics of human cognition: increasing global integration coupled with decreasing segregation found using iEEG
}

\author{
Josephine Cruzat*a , Gustavo Deco ${ }^{\mathrm{a}, \mathrm{b}, \mathrm{c}, \mathrm{d}}$, Adrià Tauste Campo ${ }^{\mathrm{a}, \mathrm{e}}$, Alessandro \\ Principe $^{\mathrm{e}}$, Albert Costa ${ }^{\mathrm{a}, \mathrm{b}}$, Morten L. Kringelbach ${ }^{\mathrm{fg}, \mathrm{h}}$ \& Rodrigo Rocamora ${ }^{\mathrm{e}}$ \\ ${ }^{\text {a }}$ Center for Brain and Cognition, Department of Information and Communication Technologies, \\ Universitat Pompeu Fabra, Ramon Trias Fargas 25-27, 08005, Barcelona, Spain \\ ${ }^{\mathrm{b}}$ Institució Catalana de la Recerca i Estudis Avançats (ICREA), Barcelona, Spain \\ ${ }^{c}$ Department of Neuropsychology, Max Planck Institute for Human Cognitive and Brain Sciences, \\ 04103 Leipzig, Germany \\ d School of Psychological Sciences, Monash University, Melbourne, Clayton VIC 3800, Australia \\ ${ }^{\text {e}}$ Epilepsy Unit, Department of Neurology, IMIM Hospital del Mar, Universitat Pompeu Fabra, \\ Passeig Marítim, 25, 08003, Barcelona, Spain. \\ ${ }^{\mathrm{f}}$ Department of Psychiatry, University of Oxford, OX3 7JX Oxford, UK \\ ${ }^{g}$ Center for Music in the Brain (MIB), Department of Clinical Medicine, Aarhus University, \\ Nørrebrogade 44, Building 10G, 8000 Aarhus, Denmark \\ ${ }^{\text {h } I n s t i t u t ~ d ' e ́ t u d e s ~ a v a n c e ́ e s ~ d e ~ P a r i s, ~ F r a n c e ~}$
}

*Corresponding author: Josephine Cruzat, Universitat Pompeu Fabra, C/ Ramon Trias Fargas 25-27, 08005 Barcelona, Spain. josephine.cruzat@upf.edu 


\section{Abstract}

Cognitive processing requires the ability to flexibly integrate and process information across large brain networks. More information is needed on how brain networks dynamically reorganize to allow such broad communication across many different brain regions in order to integrate the necessary information. Here, we use intracranial EEG to record neural activity from 12 epileptic patients while they perform three cognitive tasks in order to study how the functional connectivity changes to facilitate communication across the underlying network spanning many different brain regions. At the topological level, this facilitation is characterized by measures of integration and segregation. Across all patients, we found significant increases in integration and decreases in segregation during cognitive processing, especially in the gamma band $(50-90 \mathrm{~Hz})$. Accordingly, we also found a significantly higher level of global synchronization and functional connectivity during the execution of the cognitive tasks, again particularly in the gamma band. More importantly, we demonstrate for the first time that the modulations at the level of functional connectivity facilitating communication across the network were not caused by changes in the level of the underlying oscillations but caused by a rearrangement of the mutual synchronisation between the different nodes as proposed by the "Communication Through Coherence" Theory.

Keywords: Segregation; Integration; CTC Theory; Cognition; iEEG 


\section{Introduction}

Intracranial electroencephalography (iEEG) recordings from the human brain provide a unique opportunity to study cognitive functions measuring neural activity at the mesoscopic level. Beyond the high temporal resolution intrinsic to EEG measurements, this technique also allows also higher levels of spatial resolution and enhanced signal-tonoise ratio [1,2]. These advantages have led scientists to use the technique [3] to study several cognitive processes such as attention [4], visual perception [5, 6], language [7-9], memory [10-12], decision making [13], emotion [14, 15] and consciousness [16]. However, most of this research has attempted to assign functions to specific local brain areas by correlating task-performance with measurements of neural activity - see also recent coherence network studies [17-19]. Furthermore, most studies using iEEG have focused mainly on single-electrodes analysis, using predominantly event-related potentials [3] or spectral analysis [20].

In contrast to this regional view of brain function, growing evidence reveals that human cognition relies on the flexible integration of information widely distributed across different brain regions [21, 22]. Many studies have investigated the brain networks' properties underlying cognitive processing using EEG [23, 24], MEG [25-27] and fMRI [28-32]. In these studies cognitive processing appears to increase the global integration of information across neural networks, while at the same time leads to a decrease in the modularity of those networks $[25,30,31,33-36]$. This particular type of analysis has not been used in prior iEEG studies, nevertheless, there are other iEEG studies that have observed increased synchronization by task demands $[16,37,38]$. However, these findings have not been validated in iEEG studies yet. More importantly, there is still a lack of knowledge about the underlying mechanisms causing the cognitively driven modulation of 
the level of communication across the network, as evidenced by the corresponding changes in integration/segregation.

The "Communication Through Coherence" (CTC) theory states that the synchronisation between different neuronal populations could modulate the communication and information processing between them [39]. Indeed, two populations of neurons may communicate most effectively, when they are coherent, i.e. when their excitability level is coordinated in time. The CTC theory suggests that effective connections in a network can be shaped through phase relations, more specifically through gamma- and beta-band (30$90 \mathrm{~Hz}$ ) synchronisation, as reported experimentally [40-42]. Thus, oscillations are proposed to dynamically shape the computational role of different neuronal groups linked through static structural connectivity. Several empirical studies support task-induced changes in synchronisation at the level of individual regions during selective attention [43], working memory [44], and motor control [45]. Moreover, such task-induced changes in synchronisation have been observed between distant cortical regions during working memory [46], long-term memory encoding [47], visual attention [48], and sensorimotor integration [49]. The main aim of this paper is to link the CTC theory with modulations of the level of communication across different brain areas during human cognition. Here, we explore how cognitive processing modulates the level of integration and segregation of information in human brain networks. More specifically, we recorded iEEG data from depth electrodes stereotactically implanted for pre-surgical diagnosis in 12 epileptic patients performing three different cognitive tasks. The iEEG electrodes used a stereoelectroencephalography (SEEG) implantation methodology and covered broad regions of the brain including cortical as well as subcortical regions, so that we were able to assess the global changes at the level of a broad extended network. This allowed us to investigate the network properties using the operationally defined concepts of segregation and integration [22] as global network measures of brain function. Furthermore, we also 
assessed how these concepts relate to synchronization rather than to the oscillations level (amplitude). Finally, we would like to emphasize that our claims about the modulation of integration/segregation and the validity of CTC theory in our research are global, and it is because our statements are independent from the electrodes placement (i.e. node location, which was different for each patient) and type of cognitive processing (three different cognitive tasks were used).

\section{Materials and Methods}

\subsection{Ethics Statement}

The Clinical Research Ethical Committee of the Municipal Institute of Health Care (CEICIMAS) approved this study. Following the Declaration of Helsinki, patients were informed about the procedure and they gave their written consent before the experiment.

\subsection{Participants}

Twelve participants $(\mathrm{N}=12)$ ( 3 women; all right-handed; mean age $36.4 \pm 10.1$ years-old), evaluated for presurgical diagnosis in the Epilepsy Monitoring Unit of the Hospital del Mar (Barcelona, Spain), participated in the study. All patients were stereotactically implanted with depth electrodes for invasive presurgical diagnosis using a stereotactic ROSA robotic device (Medtech, France). The location of the electrodes was established only for clinical reasons using a SEEG approach. The implantation schemas were similar between all patients given that they were all under investigation for temporal lobe epilepsy. The number of electrodes used varied among 8 to 16 for patient with 5 to 15 contacts each (diameter: $0.8 \mathrm{~mm}$; contacts $2 \mathrm{~mm}$ long, $1.5 \mathrm{~mm}$ apart) (DixiMédical, France). All patients 
underwent an extensive neuropsychological evaluation and had normal or corrected-tonormal vision. They were within the normal range of education having completed from primary to high academic level. Table 1 summarizes personal data, pathological information and overview of implanted electrodes for each patient. Since the study aims to study the network dynamics supporting cognitive processes under normal circumstances, patients were assessed in absence of pharmacological treatment. Indeed, they were tested at least three days after the last administration of the drug.

\subsection{Cognitive Tasks}

\subsubsection{Picture-Naming Task}

In the picture-naming task, participants were asked to name 228 pictures presented in three different blocks. Pictures were black \& white line drawings of familiar objects from a wide range of semantic categories selected from the Snodgrass and Vanderwart (1980) set. Each picture appeared once centrally and sequentially on the computer screen in a pseudorandom order for $2000 \mathrm{~ms}$ followed by a fixation cross for $1000 \mathrm{~ms}$ (see Figure 1). Participants were instructed to overtly name every item as fast and accurately as possible in Spanish.

\subsubsection{Size-Judgement Task}

Participants were instructed to indicate via button press if the presented Spanish word represented an object larger than one-foot box in any dimension. Word stimuli were 68 items from two different categories: animals and man-made objects. Half of the words in each category represented objects larger than one foot. Words were auditory-presented through the computer speakers. Each word was presented sequentially in a pseudo-random order followed by an inter-trial lapse of $3500 \mathrm{~ms}$. 


\subsubsection{Lexical-Decision Task}

Participants were instructed to indicate whether the letter string presented in each trial written on a computer screen was a real word (e.g. run, table) or a pseudo word (e.g. lun, tible). We included four types of real words: motion verbs (e.g. run), static verbs (e.g. think), concrete nouns (e.g. table) and abstract nouns (e.g. theory). The task included a total of 150 trials. Each word was presented once centrally and sequentially on the computer screen in a pseudo-random order for $2000 \mathrm{~ms}$ followed by a fixation cross for $1000 \mathrm{~ms}$.

The three tasks had different stimuli presentation modality, which allowed us to compare the pattern of intracranial EEG response elicited when retrieving conceptual knowledge from a lexical form (size-judgment task) with the activity elicited when retrieving the lexical form of an object depicting a concept (picture naming task) and with the activity elicited when retrieving the concept through a written lexical form (lexical-decision task).

The three tasks were presented using the software Sketchbook Processing 2.2.1 (Programming Software, 2001 https://processing.org/) on a laptop computer at an approximate size of 5 degrees of visual angle. For the size-judgement and lexical-decision task, responses were given through a joystick connected to the computer. The accuracy of the responses for the picture-naming task was scored manually by the experimenter and for the other two tasks; the software collected response latencies and accuracy. An electronic processor "Arduino, UNO" was used to connect and synchronize both hardware; the XLTEK system with the computer (MacBook Pro). The application interfaced with an Arduino board that in turn was connected to the EEG amplifier, and at each trial a signal was sent through the Arduino to the EEG. 


\section{4 iEEG Data Acquisition and Pre-processing}

Neurophysiological responses were registered by the iEEG system from deep multichannel electrodes (DIXI Microtechniques, Besançon, France). On average, each patient had $13 \pm 2$ electrodes implanted (range 8-16) with a total of $120 \pm 13$ recording contacts (range 85127). The data were acquired continuously by the Neuroworks XLTEK system (version 6.3.0, build 636) at $32 \mathrm{kHz}$ with a headbox of 128 channels recorded at a sampling frequency of $500 \mathrm{~Hz}$ per channel. For our analysis, we only considered the channels placed in both cortex and subcortical structures. Channels placed in white matter or misplaced out of the cortex or subcortical structures were disregarded.

A bipolar montage was constituted offline to increase spatial resolution by removing any confounds from the common reference signal $[2,50]$. Bipolar signals were derived by differentiating neighbouring electrode pairs of recorded and not rejected consecutive channels within the same electrode array $[3,15,16,51]$. The continuous iEEG data was first high-pass filtered at $1 \mathrm{~Hz}$ and low-pass filtered at $150 \mathrm{~Hz}$. To remove common line contamination an extra notch filter was applied at 50 and $100 \mathrm{~Hz}$. In order to have specific spectral information, we analysed the spatio-temporal correlations of the Band Limited Power (BLP) at a given carrier frequency. This is a standard and successful approach introduced in the context of MEG analysis [52]. For that, the analysis at a given carrier frequency $f_{\text {carrier }}$ (we consider here $f_{\text {carrier }}=1$ to $130 \mathrm{~Hz}$ in steps of $4 \mathrm{~Hz}$ ) requires first that the iEEG signals are band-pass filtered within the narrow band [ $\left.\mathrm{f}_{\text {carrier }}-2, \mathrm{f}_{\text {carrier }}+2 \mathrm{~Hz}\right]$ (we used the second order Butterworth filter) and computed the corresponding envelope of each narrowband signal using the Hilbert transform $[53,54]$. The Hilbert transform yields the associated analytical signals. The analytic signal represents a narrowband signal, $\mathrm{s}(\mathrm{t})$, in the time domain as a rotating vector with an instantaneous phase, $\varphi(t)$, and an instantaneous amplitude, $A(t)$, i.e., $s(t)=A(t) \cos (\varphi(t))$. The phase and the amplitude (envelope of that carrier frequency) are given by the argument and the modulus, 
respectively, of the complex signal $z(t)$, given by $z(t)=s(t)+i . H[s(t)]$, where $i$ is the imaginary unit and $\mathrm{H}[\mathrm{s}(\mathrm{t})]$ is the Hilbert transform of $\mathrm{s}(\mathrm{t})$. We further consider only the slow components of the envelope $\mathrm{A}(\mathrm{t})$ by filtering the amplitudes again below $12 \mathrm{~Hz}$ [55]. Finally, the slow component of the envelope of each brain node, which corresponds to each bipolar channel, at a given carrier frequency was used to calculate the envelope FC (see subsection below, Data Analysis).

In addition, we ran extra simulations with a monopolar montage in order to test another possible definition of integration and to show the robustness of the results (independent of the montage). To increase spatial resolution, we removed any confounds from the common reference signal for each time point. In this case, brain nodes correspond to each single monopolar channel.

\subsection{Data Analysis}

\subsubsection{Envelope Functional Connectivity (FC)}

For the three tasks all trials were considered. The data was segmented into two windows around stimulus presentation: the first one spanning from $-500 \mathrm{~ms}$ to 0 (pre-stimulus window) from the stimulus presentation, and the second one spanning from 0 to $500 \mathrm{~ms}$ (post-stimulus windows) from the stimulus presentation. We defined an Envelope FC matrix of the continuous bipolar iEEG data for pre- and post-stimulus windows as a matrix of Pearson's correlations of the corresponding amplitude envelopes, i.e. the slow components of the BLP of iEEG signals at a given carrier frequency between two brain areas over the whole-time window for a given window (pre-stimulus and post-stimulus). Thus, the mean FC that we plot in the Results section is specific for each narrow band frequency window. 


\subsubsection{Phase-Lock Matrix}

For each time point we calculated the phase lock matrix describing the global state of synchronization across all network nodes using the bipolar montage. The elements of the phase-lock matrix are given by:

$$
\mathrm{P}_{\mathrm{ij}}(\mathrm{t})=\mathrm{e}^{-3\left|\varphi_{\mathrm{i}}(\mathrm{t})-\varphi_{\mathrm{j}}(\mathrm{t})\right|}
$$

where $\varphi_{\mathrm{i}}(\mathrm{t})$ is the extracted phase of node $i$ at time $t$ (at a given carrier frequency using the Hilbert transform as specified above). One can use the phase-lock matrix at a specific single time point to calculate the integration as specified below. For the other simulation, where we contrast the pre- and post-stimulus windows, we considered the averaged phaselock matrix across the time elapsed in the respective windows.

\subsubsection{Integration}

We used the measure of integration introduced by Deco et al. (2015), defined at the network level, to characterize the level of broadness of communication between regions across the whole brain. First, we filtered the data, and then we calculated the envelope FC as explained above for both pre- and post-stimulus windows. We define integration as the size of the largest connected component in the FC matrix. That is, the number of nodes of the largest connected graph in the binarized functional connectivity matrix obtained after thresholding. More specifically, for a given absolute threshold $\theta$ between 0 and 1 (scanning the whole range), the $\mathrm{FC}$ (using the criteria $\left|\mathrm{FC}_{\mathrm{ij}}\right|<\theta$, i.e a value of 0 and 1 otherwise) can be binarized and the largest subcomponent (see definition below) extracted as a measure of integration. To get a measure that is independent of the threshold, this curve can be integrated in the range of thresholds between 0 and 1 . This integration measure is normalized by the maximal number of connected brain areas (that is, all $\mathrm{N}$ areas) for each integration step and by the number of integration steps such that the maximal integration is 
normalized to 1 .

Note, that the concept of integration was designed for considering the broadness of communication across the brain, and thus required us to consider positive and negative correlations as potentially establishing a communication link. Therefore, we took the absolute values of the FC correlations. In other words, we quantified the broadness of communication (measured by means of correlation) regardless of whether the correlation values were negative or positive.

In graph-theoretical terms, subcomponents are extracted from the undirected graph defined by the binarized matrix (which itself is considered as an adjacency matrix). More precisely, a subcomponent is a subgraph in which paths connect any two vertices to each other, and which connects to no additional vertices in the super-graph[22]. A vertex $u$ is said to be connected to a vertex $v$ in a graph $G$ if there is a path in $G$ from $u$ to $v$. The concepts of subgraph and super-graph are defined as following: Let $H$ be a graph with vertex set $V(H)$ and edge set $E(H)$, and similarly let $G$ be a graph with vertex set $V(G)$ and edge set $E(G)$. Then, we say that $H$ is a subgraph of $G$ if $V(H) \subseteq V(G)$ and $E(H) \subseteq E(G)$. In such a case, we also say that $G$ is a super-graph of $H$.

\subsubsection{Segregation}

As a complement of the integration, we used the modularity measure [56] as a measure of segregation. Following Rubinov and Sporns (2011), modularity is defined as a measure of the goodness with which a network is optimally partitioned into functional subgroups, i.e. a complete subdivision of the network into non-overlapping modules, and supported by densely connected network communities. We consider the modularity of our envelope FC matrix. This matrix contains positive and negative weights, namely the corresponding correlation between two nodes. Our measure of modularity is given by, 


$$
Q^{G J A}=\frac{1}{v^{+}+v^{-}} \sum_{i j}\left[\left(w_{i j}^{+}-e_{i j}^{+}\right)-\left(w_{i j}^{-}-e_{i j}^{-}\right)\right] \delta_{M_{i} M_{j}}
$$

Where the total weight, $\mathrm{v}^{ \pm}=\sum_{\mathrm{ij}} \mathrm{W}_{\mathrm{ij}}^{ \pm}$, is the sum of all positive or negative connection weights (counted twice for each connection), being $\mathrm{w}_{\mathrm{ij}}{ }^{+} \in(0,1]$ the weighted connection

between nodes $\mathrm{i}$ and $\mathrm{j}$. The chance-expected within-module connection weights $e_{i j}^{ \pm}=\frac{s_{i}^{ \pm} s_{j}^{ \pm}}{v^{ \pm}}$ , where the strength of node $\mathrm{i}, \mathrm{s}_{\mathrm{i}}^{ \pm}=\sum_{\mathrm{j}} \mathrm{W}_{\mathrm{ij}}{ }^{ \pm}$, is the sum of positive or negative connection weights of $i$. The delta $\delta_{\text {MiMj }}=1$ when $i$ and $j$ are in the same module and $\delta_{\text {MiMj }}=0$ otherwise [57]. This definition is a generalisation of the standard measure of modularity for matrices with nonnegative weights, which is given by the average difference between present within-module connection weights $\mathrm{w}_{\mathrm{ij}}{ }^{+}$and chance-expected within-module connection weights $\mathrm{e}_{\mathrm{ij}}{ }^{+}$. As mentioned above, here we consider both positively and negatively weighted connections (envelope FC matrix). The positively weighted connections represent correlated activation patterns and hence reinforce the placement of positively connected pairs of nodes in the same module. The negatively weighted connections represent anti-correlated activation patterns and reinforce the placement of negatively connected pairs of nodes in distinct modules. For a complete description see [58].

\subsubsection{Synchronization}

We measure the global mean level of synchronization as the mean value of the Kuramoto order parameter across time. The Kuramoto order parameter is defined by following equation:

$$
\left.R(t)=\mid \sum_{k=1}^{n} e^{(i \varphi}(t)\right) \mid / n
$$


where $\varphi_{\mathrm{k}}(\mathrm{t})$ is the instantaneous phase of each narrowband signal at node $\mathrm{k}$ at a given carrier frequency by using the Hilbert derived phases of the slow component of the Band Limited Power (BLP) signals. The Kuramoto order parameter measures the global level of synchronization of the $\mathrm{n}$ oscillating signals. Under complete independence, the $n$ phases are uniformly distributed and thus $R$ is nearly zero, whereas for $\mathrm{R}=1$ all phases are equal (full synchronization).

\subsubsection{Permutation Tests}

To examine the reliability of the post-stimulus window modulation, frequency-dependent statistical significance was assessed using a non-parametric test with 1000 random permutations. The test statistic was chosen to be the median across the differences between post and pre-stimulus measures. Following Winkler et al. (2014), we simulated the null hypothesis of no stimulus modulation at any post-stimulus windows by randomly permuting pre- and post-stimulus samples at each paired trial (group exchangeability hypothesis) and computing the test statistic. These surrogate values formed a reference distribution, against which we compared the original statistic value. The proportion of permutations in which surrogate values matched or exceeded the original statistic value determined the test $\mathrm{p}$-value $(\mathrm{P})$ to be compared with the significance level. We obtained a p-value for each frequency band and applied multiple comparisons corrections with the False Discovery Rate (FDR) level 0.05 using the Benjamini-Hochberg method. 


\section{Results}

In the present study, we exploited the high spatiotemporal resolution of intracranial electroencephalography (iEEG) recordings to investigate the reorganization of brain networks driven by cognitive tasks. This technique is usually employed in pharmacologically resistant epileptic patients $[59,60]$ who require brain mapping before surgery, and is also a useful tool for basic research on cognitive neuroscience [3].

We recorded iEEG data in twelve patients while performing a picture-naming task (Figure 1A). This task was used to drive the modulation of the underlying brain networks related to the integration of task-related information. It is known that for the large majority of people language is supported by a widespread large-scale network distributed across frontal, temporal, parietal and occipital lobes in the dominant hemisphere [29, 61, 62]. During the task, patients had to overtly name each picture as fast as possible in Spanish (see Materials and Methods). The picture naming accuracy was high (83.2 $\pm 11.3 \%)$ (Figure 1B) with an averaged response time of $1350 \pm 306 \mathrm{~ms}$. All channel recordings from grey matter and subcortical structures were considered for the analysis. Although neural activity signal was recorded from all lobes in both hemispheres, most of the recordings were consistently obtained from the left frontal, temporal and parietal lobes (see an example of implantation scheme in Figure 1C). After pre-processing the data, we analysed the Band Limited Power (BLP) at a given carrier frequency $\left(f_{\text {carrier }}\right)$ in order to have specific spectral information. We band-pass filtered the iEEG signals within the narrow band $\left[f_{\text {carrier }}-2, f_{\text {carrier }}+2 \mathrm{~Hz}\right]$ and considered a range of $f_{\text {carrier }}$ starting from 1 to $130 \mathrm{~Hz}$ in steps of $4 \mathrm{~Hz}$. We chose a bandwidth of $4 \mathrm{~Hz}$ because it provides a good trade-off between phase estimation accuracy and number of testable comparisons. In order to compute the envelope Functional Connectivity (FC), we further computed the Hilbert transform (Figure 2). To study 
changes in the functional network topology during the execution of a cognitive task, we contrasted two situations, namely: a pre-stimulus window of $500 \mathrm{~ms}$ before the picture was presented: "pre-stimulus window", and a post-stimulus window of $500 \mathrm{~ms}$ immediately after the picture was presented: "post-stimulus window". As speech production is associated with large motor activity, thus with a significant increase of integration, we focused our analysis in a time window far from the beginning of the motoric activity (around $1350 \pm 306 \mathrm{~ms}$ ). In order to characterize the organization of the network under both situations, we used the integration and segregation measures of global brain function (see Materials and Methods) characterizing the level of communication across the different nodes of the brain network. We tested the statistical significance of the network measure differences between pre-stimulus and post-stimulus using a non-parametric method [63]. Specifically, we characterized the null hypothesis using constrained permutations that preserved the trial grouping of the data at both time windows (Materials and Methods).

The main results come from the comparison of the integration and segregation measures during the pre- and post-stimulus windows. Here, we first focus on a single patient to illustrate the main results (Figure 3). Neural integration significantly increases during cognitive processing (Figure 3A) and complementary to this measure, we observed a consistent decrease in segregation as measured by the modularity (Figure 3B). In both cases, the greatest modulation appeared particularly in the gamma band, around 50 to 90 $\mathrm{Hz}(\mathrm{p}<0.05, \mathrm{~N}=1000)$. Note that the concepts of integration and segregation are measures that by definition are calculated independently from each other but their results are highly correlated; when one increases the other consistently decrease.

Having found an increase in the integration consistently associated with a decrease in the segregation, the question then arises what is behind the modulation of these measures driven by the task. We first examined whether these modulations could be explained by changes in the oscillations' envelope amplitude. Importantly this was not the case; no 
modulation of oscillation amplitude at any frequency (Figure 3C) could explain either the integration increases or the modularity decreases. This occurred both when looking at the mean amplitude of all electrodes together and when evaluating the mean amplitude of each bipolar channel separately at $60 \mathrm{~Hz}$ (which corresponds to the maximal modulation of the integration measure) (Figure 3F).

Further, we looked whether the level of global synchronization of the corresponding envelopes was associated with the modulation of the integration and segregation. We used the Kuramoto Order Parameter (see Materials and Methods) to measure the mean synchronization of cortical activity under both conditions. We observed that, in fact, there is an increase of mean synchronization over a broad range of frequencies that is greater in the gamma range (50 to $90 \mathrm{~Hz}$ ) for the post-stimulus window (Figure 3D). Next, we computed the Envelope Functional Connectivity (see Materials and Methods) between channels to see whether it behaves coherently with the level of global synchronization. We calculated it through the instant amplitude envelopes of the given frequencies. The analysis revealed that the Envelopes' Functional Connectivity is enhanced during task performance, in particular in the gamma range (50 to $90 \mathrm{~Hz}$ ) (Figure 3E).

Together these results suggest that given that the relationship between integration and segregation cannot be accounted by an increase of the oscillations, the most likely explanation for these phenomena is a global increase of the connectivity, especially in the gamma band. This is consistent with the notion that communication between different brain networks is accomplished by means of the level of synchronization as posited by the Communication Through Coherence Theory (CTC) [40, 42].

Importantly, the significant integration and segregation modulations driven by the stimulus presentation were consistently found in every patient. Figures 4 and 5 plot for each single patient across windows, the cognitive modulation of integration and segregation, 
respectively. As shown in the figures, despite the heterogeneity of the recording sites, all patients consistently show the same pattern of modulation. Figure 6 shows the group average data for both measures.

The increase of integration and decrease of segregation were highly correlated as it is shown quantitatively by the Pearson correlation of -0.9846 ( $\mathrm{N}=12$ patients) of the respective modulations at the carrier frequency of $60 \mathrm{~Hz}$ (maximal modulatory effect point). Figure 7 shows the correlation between both measures.

Interestingly, in most of the patients, there was a consistent decrease of the segregation in the gamma band but with a slight increase of the segregation in the sub-gamma regime. This is not observed for the integration measure. Figure 8 shows the oscillations' envelope amplitude at $60 \mathrm{~Hz}$ for each bipolar channel and for each patient. In all cases, the amplitude of the oscillations envelope across electrodes does not differ between pre- and post-stimulus windows. Indeed, the majority of the electrodes (over 98.6\%) do not show significant differences in amplitude modulation.

In order to uncover if the integration/segregation modulations could solely be linked to one particular task, we analysed two other cognitive tasks carried out by the patients, namely: 1) a lexical decision task; and 2) a size judgement task (see Materials and Methods). The performance in both tasks was high $(81.8 \pm 5.1 \%$ and $88.7 \pm 8.3 \%$ respectively $)$. The averaged response time for the lexical decision task was $1481 \pm 398 \mathrm{~ms}$ and for the size judgement task $1008 \pm 287 \mathrm{~ms}$. Similar to the results for the picture-naming task, we found a significant modulation of the integration in the post-stimulus window (Figure 9A and 9D). In addition, as shown in Figure 9B and 9E we found corresponding consistent significant decreases in segregation. Consistently with the picture-naming task, the largest modulation appears in the gamma band (around 50 to $90 \mathrm{~Hz}$ ) for both measures. 
Consequently, the envelopes mean amplitude did not change in response to the stimulus presentation (Figure 9C and 9F).

Next, we investigated whether the integration modulation was related to the performance. Interestingly, we found that this was not the case as shown by a Pearson correlation of $0.0242(\mathrm{~N}=12$ patients $)$ between the accuracy and the integration measurement in the poststimulus window.

Further, to verify that the increases in integration and decreases in segregation measures are indeed task-driven, we contrasted the same measurements in task absence conditions. Figure 10 shows (in one participant) the lack of integration (Figure 10A) and segregation (Figure 10B) modulation when we contrasted random $50 \%$ of pre-stimulus windows trials against the other $50 \%$ (same windowing as before, i.e. $500 \mathrm{~ms}$ before stimulus presentation).

In order to check that the results are not dependent on the montage, we computed the integration and segregation measurements by also using the monopolar montage. First, we compared the new integration/segregation measurements with the more classical approach considering the pairwise changes in the FC between the post- and pre-stimulus windows. Figure $11 \mathrm{~A}$ and $11 \mathrm{~B}$ plots for a single patient the integration and segregation modulation during the pre- and post-stimulus windows, but now based on the monopolar montage. The results are consistent with the previous analysis and confirm the robustness of the findings. We also show in Figures 11C-E the FC matrices for a specific frequency $(60 \mathrm{~Hz}$, which is where we observed the greatest modulation across all patients and tasks) for pre- and poststimulus windows and their differences respectively. The differences between both matrices (Figure 11E) demonstrate the distributed character of the modulation across many different nodes (which corresponds to each channel). This fact suggests that the cognitivedriven global changes in integration and modulation are indeed not associated with any 
particular anatomical position inside the networks considered. These results were found consistently for all participants as shown in Figure S1 in the Supplementary Materials.

Furthermore, Figure 12 shows that there is no correlation between the integration measure and the mean changes in the FC between the post- and pre-stimulus windows, for different frequencies in the Gamma range. The lack of correlations demonstrates that our measure of integration differs and complements the more classical measure of pairwise correlation.

Still, the envelope FC do not necessarily reflect communication. To explicitly confirm the role of the phase coupling in the task-driven modulation of integration and segregation, we extended the analysis of those measures by using the phase-lock matrix (as a more explicit measure of communication as expressed by the CTC theory). The phase-lock matrix (see Materials and Methods) directly characterizes the global state of mutual synchronization between all pairs of network nodes. Further, in this context we are able to study the temporal evolution of the integration across time in small sliding windows. For this purpose, we adapted the concept of integration to the phase lock matrix which can be defined for each time point (given that is defined by the instantaneous phases of the iEEG signal), and thus it is more robust for small windows (even for instantaneous single time points). In other words, for each single time point, we computed the phase-lock matrix (see Material and Methods) and the corresponding integration measure. Figure 13 plots the evolution of this integration measure for the whole period of time (from $1000 \mathrm{~ms}$ before task onset to $1500 \mathrm{~ms}$ after task onset) at $80 \mathrm{~Hz}$. The figure shows a monotonic increasing behaviour of the integration starting after the onset of the task. This observation is relevant because it suggests that the integration is not only driven by the stimuli onset but is persistent in time. This result was found in all tasks.

Although the integration is by definition a global measurement, we investigated whether the cognition-driven modulations of the integration vary for different subnetworks. Given 
that for two patients we had precise anatomical information about the location of the channels, we were able to perform the integration analysis restricted to three different specific subnetworks, namely, the frontal, temporal and parietal subnetworks. Figure 14 shows the cognitive driven modulation of the integration for these two patients and for the three different subnetworks. The figure demonstrates that even though the greatest modulations were found in both right and left temporal lobe, the parietal and frontal subnetwork also show a statistical significant modulation. This suggests the global character of the modulation of the broadcasting of communication across the brain due to cognitive processing.

\section{Discussion}

We used intracranial EEG (iEEG) to record human neural activity from 12 epileptic patients while they were performing three different tasks (picture-naming, lexical decision and size judgement) in order to study how cognition modulates global functional network measurements, namely integration and segregation. Across all patients and all tasks, we found a significant increase in integration and decrease in segregation during cognitive processing $(\mathrm{p}<0.05, \mathrm{~N}=1000)$, especially in the gamma band $(50-90 \mathrm{~Hz})$. The modulations of integration and segregation were highly inversely correlated, independent of the montage used, driven by the stimuli and were not driven by changes in the underlying level of oscillations given that the oscillations' amplitude of pre- and post-stimulus windows were not significantly different. Instead, we found significantly higher level of synchronisation (as measured using the Kuramoto order parameter) and functional connectivity during the tasks, again particularly in the gamma band. These results suggest that the cognitive modulation of the broadness of communication across the entire 
extended network could be due to a rearrangement of the coherence level between the nodes, which would fit the predictions of the CTC theory $[40,42]$ extended to the wholebrain level [39]. The CTC hypothesis posits that the mechanism through which information is transmitted is by the synchronization of distinct neuronal populations, mainly in the gamma and beta-band $(30-90 \mathrm{~Hz})[39]$. Note that the original CTC theory is based on a phase synchronization mechanism defined at the neuronal level. Here, as proposed by Deco \& Kringelbach [39], we extend the hypothesis to the mesoscopic/macroscopic level by analysing the phases and correlations of the envelopes of the Band Limited Power.

In our study we used global measurements, i.e. quantities like the level of integration and segregation that describe the global level of reorganization of the dynamics during the performance of a cognitive task. The rationale behind this election is twofold: 1) the integration and segregation measurements describes directly the level of broadcasting of communication across the brain driven by cognition, and 2) global measurements are particularly well suited for cases where the nodes considered are different for each subject.

Traditionally, graph and information theoretical approaches have helped to characterize the global network connectivity in terms of segregation and integration in the brain $[22,64-$ 66]. Segregation refers to the relative statistical independence of subsets of neurons or brain regions to compute information $[64,66]$, while integration is a complementary concept quantifying the level of connectivity across the whole-brain $[22,66]$. In particular, we used a measure of integration based on the largest component present in the wholebrain connectivity matrix [22], while for the segregation we used the concept of modularity $[56]$.

Prior studies on reconfiguration of functional networks have analysed different cognitive states solely by the concept of functional connectivity $[28,30,32,33]$. These studies have revealed that in the human brain local computations are likely to be highly segregated in 
spatially distributed network communities with clustered connectivity [67]. Alternatively, integration (and segregation) can be consistently defined by characterizing with the mutual information the overall deviation from statistical independence across a set of nodes [68]. Further measures of functional integration and segregation include neural complexity (which is defined as the coexistence of functional segregation and integration within the same network [64]), and integrated information (which is defined as the mutual information across the weakest partition of a system [69-71]). In comparison but complementary to these variety of functional measurements, here we use a novel concept introduced by Deco et al. [22], which associates integration with the broadness of communication across the whole brain quantified by the length of the largest connected component of the functional connectivity.

It is rather clear that the integration measure used in the present study and the functional connectivity measure of band-limited power are not correlated. These uncorrelated values suggest that the functional connectivity per se is not giving explicit information of how clustered it is the network. Indeed, one can have higher correlations but a lot of functional segregated clusters which in our measure is associated with low integration.

Our results support the view of the brain as a complex system organized into large-scale networks. In order to support cognitive functions, these networks need to flexibly adjust their functional connectivity to integrate relevant information towards a goal-directed behaviour. This findings are in line with previous neuroimaging studies showing increases of the integration across brain networks as evidenced by MEG during working memory processing [25, 36], MRI during emotional and motivational stimulus processing [31] and fMRI for selective attention [72].

We found that there is a decrease in segregation in the gamma band with task, yet an increase in segregation in the sub-gamma band. The decrease of the segregation in the 
gamma regime is expected and consistent with the increase of integration in the same regime which is putatively linked to increased stimuli/cognitive processing [42]. Along the same lines of argument, the increase of segregation in the sub-gamma bands could perhaps be associated with a disengagement of large slow resting networks. It would be of considerable interest to further investigate.

It is important to remark the fact that the implantation scheme of the electrodes is different for each patient. This works to our advantage, since the main aim was to show whether there is an increase of integration (i.e. increases in the level of connectivity across the whole network) across the whole-brain, and thus independent of both, the positions of individual electrodes and the montage. Further strengthening the results, we analysed three different cognitive tasks, which demonstrated that the main modulatory effect on the integration/segregation and the validity of the CTC theory was independent of the particular cognitive processing. Thus, because of our global perspective, unlike many previous studies, we did not investigate the role of single channels in the different cognitive processes. Nevertheless, we are convinced that localising these electrodes in the human brain could lead to the discovery of interesting differences at the local spatial level and that this could be a very interesting goal for future investigations.

In our study, we did not find a correlation between the performance and the modulation of the integration. Although we cannot presently elucidate the dynamical properties that leads to a better performance, the present findings suggest that the behavioural errors in our experiment are not captured in the time window we were looking at, or were not due to a failure at the global level of communication, but probably caused by failures at the local level of specific processing.

Equally, it would be interesting to examine in future investigations how the integration and segregation measures might be differently modulated under different cognitive load and as 
a function of the task were performed correctly or erroneously. An important future goal would be to go beyond global measures to determine specifically which brain areas or local networks show the highest degree of task-driven effective connectivity and therefore are mostly involved in information processing. In this context, future studies could greatly benefit from diffusion tensor imaging in two ways: to visualize and describe the precise connectivity between the electrodes in the brain, as well as the basis of whole-brain models considering the connectivity and continuity of neural pathways in the patients.

There are potential methodological limitations due to the fact that iEEG in humans is always obtained from epileptic patients who, besides having epileptogenic neural activity, may have also differences in the structural connectivity. The lack of whole-brain coverage with this technique means that we have to be cautious when making assumptions of global network connectivity because of the restriction of the spatial coverage that can be simultaneously sampled. In this study, although patients suffered from Temporal Lobe Epilepsy, most of the electrodes were implanted in many parts of the frontal, temporal and parietal lobes. Therefore, the cognitive-driven changes observed on the level of integration and segregation are mainly from these areas. Nevertheless, we speculate that the effect is probably global across the whole brain. For that, we predict that those changes could be consistently observed in fMRI and/or EEG/MEG experiments.

\section{Conclusions}

We show that cognitive processing drives a global increase of integration and decrease of segregation, especially in the gamma band $(50-90 \mathrm{~Hz})$, which could reflect broadcasting of communication. The results add to the emerging interesting literature on integration and 
segregation in the human brain (see [73] for a careful review). More importantly, we demonstrate here for the first time that these modulations in the level of functional network topology were not caused by changes in the level of the underlying oscillations but caused by a rearrangement of the band-limited functional connectivity between the different nodes, according to the "Communication Through Coherence" Theory.

\section{Acknowledgements}

The authors would like to thank all patients for their participation and the IMIM-Hospital del Mar Epilepsy Unit staff for their technical assistance in collecting the data. This work was supported by Gustavo Deco's ERC Advanced Grant: DYSTRUCTURE (n. 295129), from the European Union's Horizon 2020 research and innovation programme under grant agreement No. 720270 and by the Spanish Research Project PSI2013-42091-P. M.L.K. is supported by the ERC Consolidator Grant CAREGIVING (n. 615539) and the Center for Music in the Brain, funded by the Danish National Research Foundation (DNRF117). 


\section{Table and Figures}

Table 1. Demographic and clinical characteristics of each patient.

\begin{tabular}{|c|c|c|c|c|c|c|c|c|}
\hline Patient & Gender & $\begin{array}{c}\text { Age } \\
\text { (years) }\end{array}$ & $\begin{array}{c}\text { Epileptogenic } \\
\text { zone } \\
\text { laterality }\end{array}$ & $\begin{array}{l}\text { Seizure onset } \\
\text { zone }\end{array}$ & $\begin{array}{l}\text { Implanted } \\
\text { Regions }\end{array}$ & $\begin{array}{c}\mathbf{N}^{\circ} \text { of } \\
\text { electrodes } \\
\text { implanted }\end{array}$ & $\begin{array}{c}\mathrm{N}^{\mathbf{0}} \\
\text { unipolar } \\
\text { channels }\end{array}$ & $\begin{array}{c}\mathrm{N}^{\circ} \text { of } \\
\text { bipolar } \\
\text { channels } \\
\text { for analysis }\end{array}$ \\
\hline A & Male & 38 & Left & $\begin{array}{l}\text { Mesial temporal } \\
\text { and amygdala }\end{array}$ & L (F-T-I-P) & 8 & 85 & 76 \\
\hline B & Male & 21 & Left & Anterior temporal & $\begin{array}{c}\mathrm{L}(\mathrm{F}-\mathrm{T}-\mathrm{I}-\mathrm{P}-\mathrm{O}) \& \\
\mathrm{R}(\mathrm{F}-\mathrm{T})\end{array}$ & 15 & 125 & 95 \\
\hline $\mathrm{C}$ & Male & 44 & Right & Anterior temporal & $\mathrm{L}(\mathrm{T}) \& \mathrm{R}(\mathrm{F}-\mathrm{T}-\mathrm{P})$ & 12 & 125 & 104 \\
\hline $\mathrm{D}$ & Male & 44 & Right & Temporo-parietal & $\begin{array}{c}\mathrm{L}(\mathrm{T}) \& \mathrm{R}(\mathrm{F}-\mathrm{T}-\mathrm{I}- \\
\mathrm{P})\end{array}$ & 16 & 127 & 101 \\
\hline $\mathrm{E}$ & Female & 43 & Left & $\begin{array}{l}\text { Anterior medial } \\
\text { temporal }\end{array}$ & L (F-T-I-P-O) & 11 & 127 & 112 \\
\hline $\mathrm{F}$ & Male & 25 & Right & $\begin{array}{c}\text { Temporo-parieto } \\
\text { occipital }\end{array}$ & R (F-T-I-P-O) & 14 & 127 & 105 \\
\hline G & Male & 23 & Left & Temporal & L (F-T-I-P) & 13 & 125 & 102 \\
\hline $\mathrm{H}$ & Female & 43 & Left & $\begin{array}{l}\text { Posterior } \\
\text { Temporal }\end{array}$ & L (F-T-I-P) & 14 & 125 & 103 \\
\hline I & Male & 44 & Left & Mesial Temporal & L (F-T-I-P) & 12 & 123 & 105 \\
\hline $\mathrm{J}$ & Female & 46 & Left & $\begin{array}{l}\text { Anterior medial } \\
\text { temporal }\end{array}$ & L (F-T-I-P-O) & 11 & 124 & 107 \\
\hline $\mathrm{K}$ & Male & 43 & Left & Mesial Temporal & L (F-T-P) & 9 & 103 & 94 \\
\hline $\mathrm{L}$ & Male & 23 & Right & Anterior temporal & $\mathrm{R}(\mathrm{F}-\mathrm{T}-\mathrm{P})$ & 15 & 125 & 96 \\
\hline
\end{tabular}

R: Right; L: Left; F: Frontal; T: Temporal; P: Parietal; O: Occipital; I: Insula 
A.

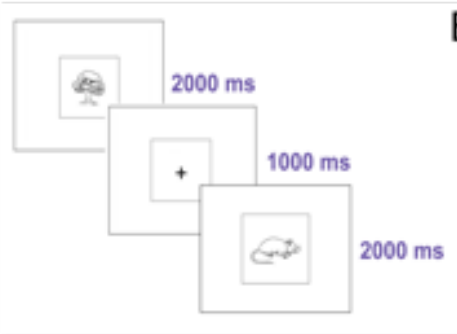

B.

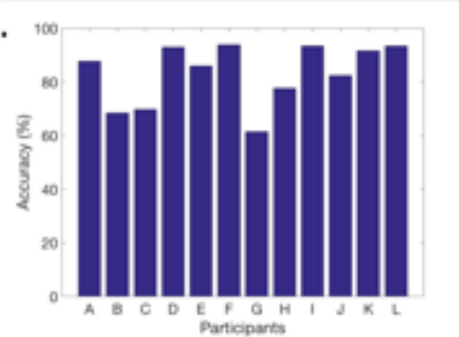

C.

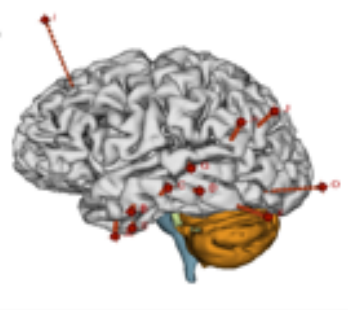

Figure 1. Paradigm, behavioural performance and implantation scheme. A) Schematic example of the experimental paradigm of the picture-naming task. After an interval of $1000 \mathrm{~ms}$ for preparation, each target picture was presented and remained on the screen for 2000 ms. B) Picture naming accuracy for each patient. On average, patients achieved $83.2 \pm 11.3 \%$ accuracy on test items $(N=228)$. C) Example of the intracerebral implantation scheme for iEEG recordings in patient E. Eleven electrodes were implanted in the left hemisphere. 


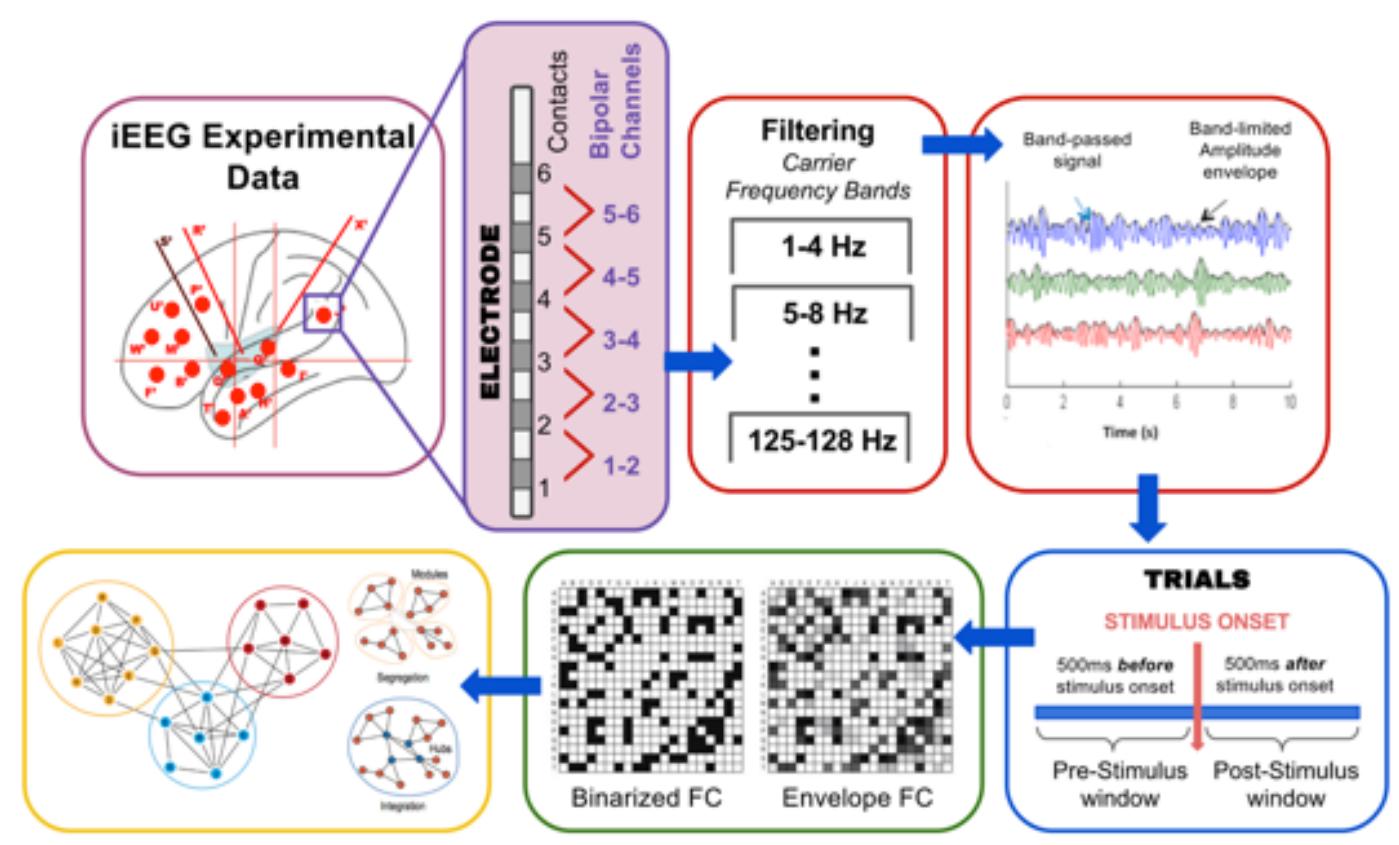

Figure 2. Data processing flow chart. The iEEG data is recorded from 85 up to 127 unipolar channels on each patient. The bipolar montage is constituted offline by subtracting the neural activity recorded by neighbouring contacts within the same electrode array. The data is first band-pass filtered at 1 to $150 \mathrm{~Hz}$, and further band-pass filtered into narrow frequency bands $\left[f_{\text {carrier }}-2, f_{\text {carrier }}+2 \mathrm{~Hz}\right]$ (we consider here carrier frequencies $f_{\text {carrier }}=1$ to $130 \mathrm{~Hz}$ in steps of $4 \mathrm{~Hz}$ ). By the Hilbert transform the corresponding amplitude envelopes are computed to further compute the envelope FC matrix. The continuous data is segmented into windows of -500 to $0 \mathrm{~ms}$ (pre-stimulus window) and 0 to $500 \mathrm{~ms}$ (post-stimulus window), around stimulus presentation. In order to characterize the organization of the network under both windows, we used the integration and segregation measures of global brain function. 

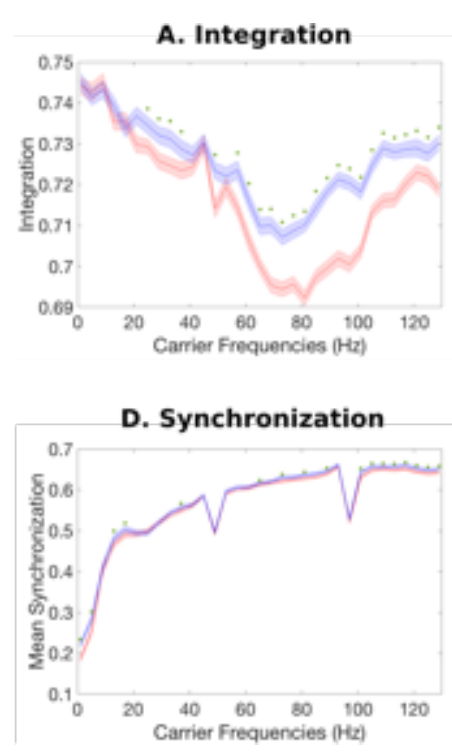

B. Segregation

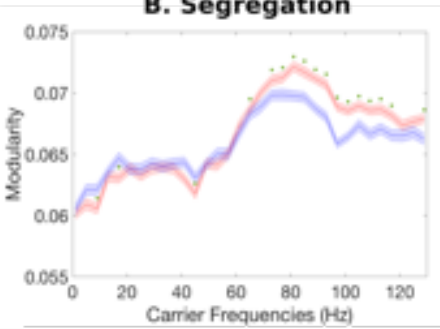

E. Functional Connectivity

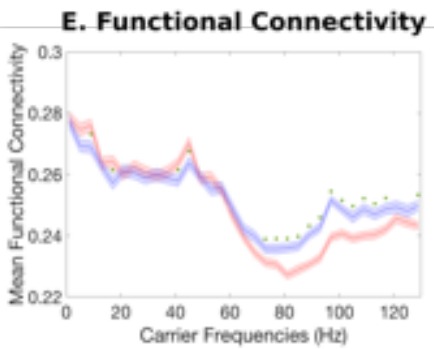

C. Envelopes' Amplitude

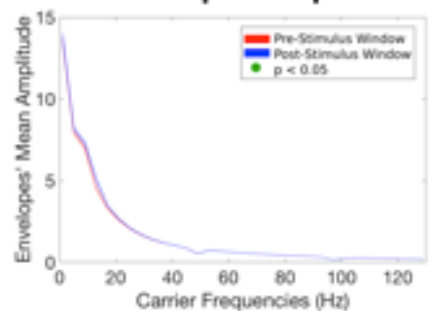

F. Envelopes' Amplitude

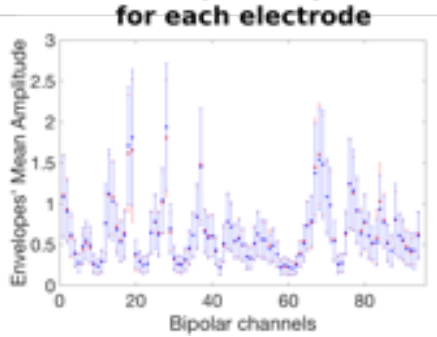

Figure 3. Results analysis patient $\mathrm{K}$ for the picture-naming task. Panel A shows a significant increase in the integration during cognitive processing. The greatest effect is observed in the gamma range (50 to $90 \mathrm{~Hz}$ ). The red line corresponds to pre-stimulus window, the blue line corresponds to post-stimulus window, the shaded error regions reflect the standard deviation across trials and green dots indicate a statistical significance of $p<0.05(N=1000)$. Complementary to the integration, panel $B$ shows a decrease of the modularity in the same frequency range. Panel $C$ shows that there are no iEEG oscillations amplitude changes in any frequency induced by the stimulus (both curves are strongly overlapped). This result indicates that the increase of integration and decrease of modularity could not be explained by changes in the oscillations amplitude. Panel D shows an increase of mean synchronization over a broad range of frequencies that is more conspicuous in the gamma band range (50 to $90 \mathrm{~Hz}$ ) for the post-stimulus window. Panel E shows that the functional connectivity behaves coherently with the other results, as it increases as a function of the stimulus presentation particularly in the gamma range. Panel $F$ plots the amplitude of the oscillations envelope at $60 \mathrm{~Hz}$ for each bipolar channel and pre-and post-stimulus window. There are no noticeable modulations across single bipolar channels between pre-and post-stimulus window. Note that the sharp peaks at 50 and $100 \mathrm{~Hz}$ are due to the power-line noise created by the electrical power. 


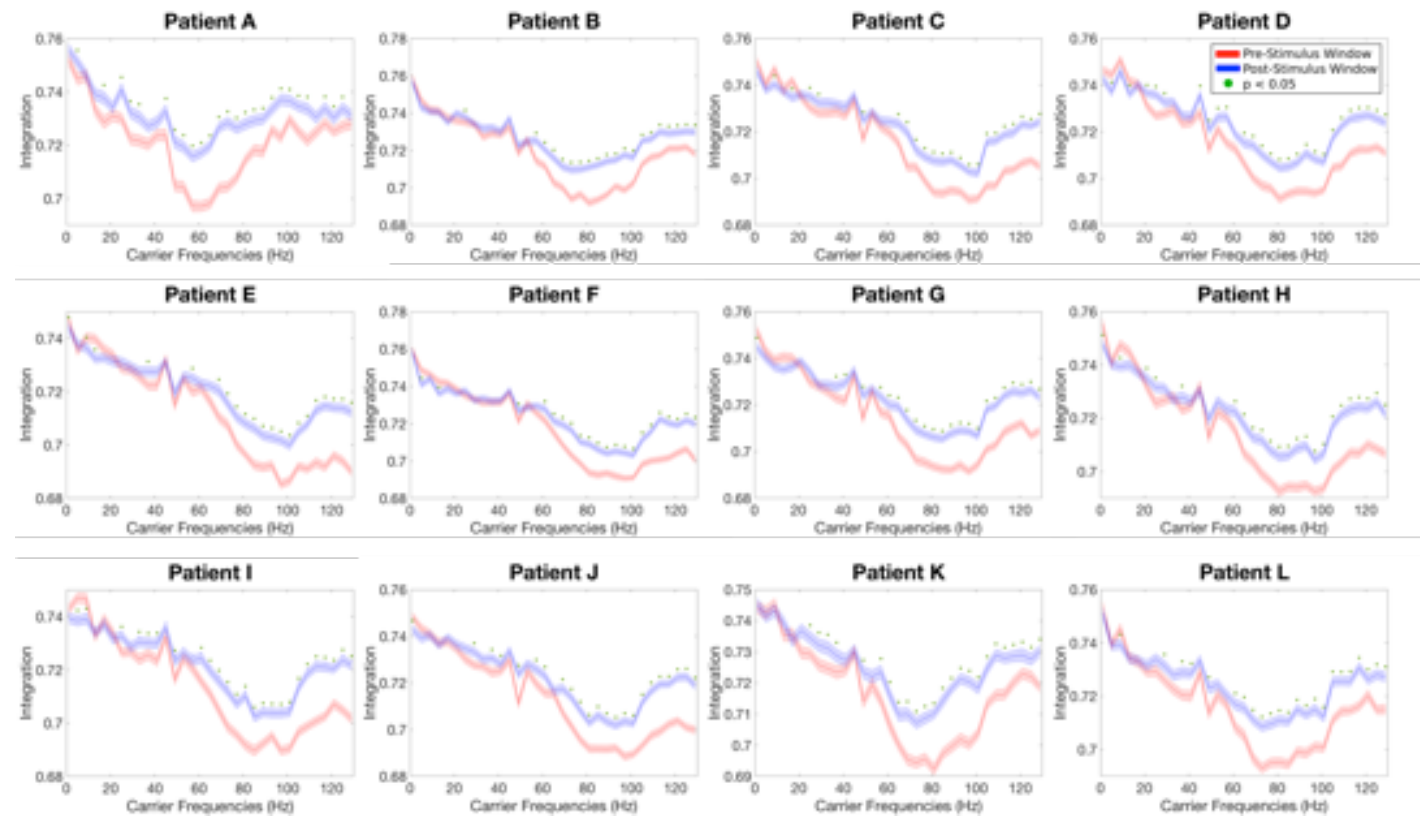

Figure 4. Integration measure results for each patient. The panels show the results for every single patient for the picture-naming task. As can be seen, despite the heterogeneity of the recording sites, all patients show a significant increase of the integration related to cognitive processing. For all patients, the greatest effect was found in the gamma range. The red line corresponds to pre-stimulus window, blue line corresponds to task condition, the shaded error regions reflect the standard deviation across trials and green dots indicates a statistical significance of $p<0.05(N=1000)$. 

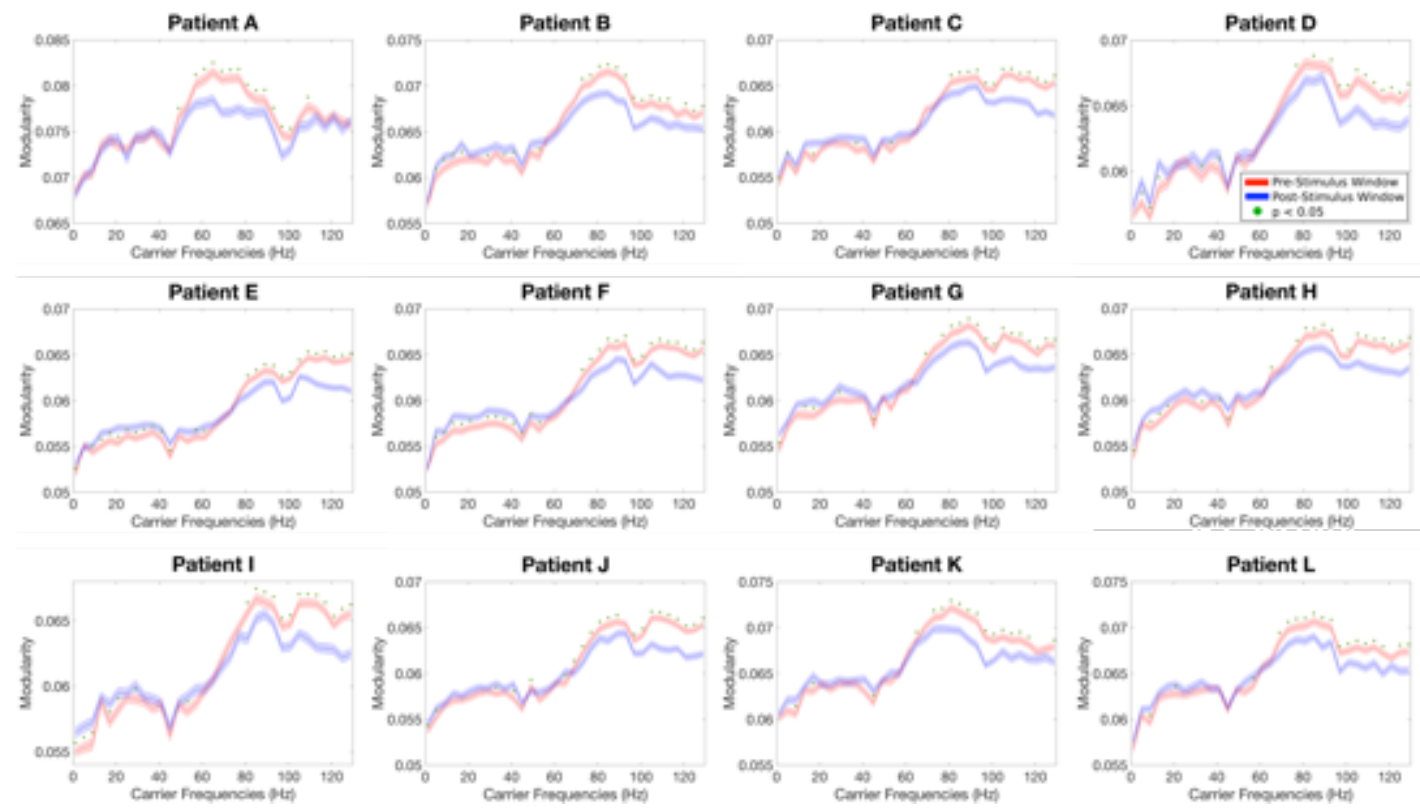

Figure 5. Segregation measure results for each patient. The panels show the results of segregation (measured by the modularity) during pre-and post-stimulus window for the picture-naming task. For all patients, there is a significant decrease of the segregation during cognitive processing and the greatest effect can be seen in the gamma range (50 to $90 \mathrm{~Hz}$ ). The red line corresponds to pre-stimulus window, blue line corresponds to poststimulus window, the shaded error regions reflect the standard deviation across trials and green dots indicates a statistical significance of $p<0.05(N=1000)$. 

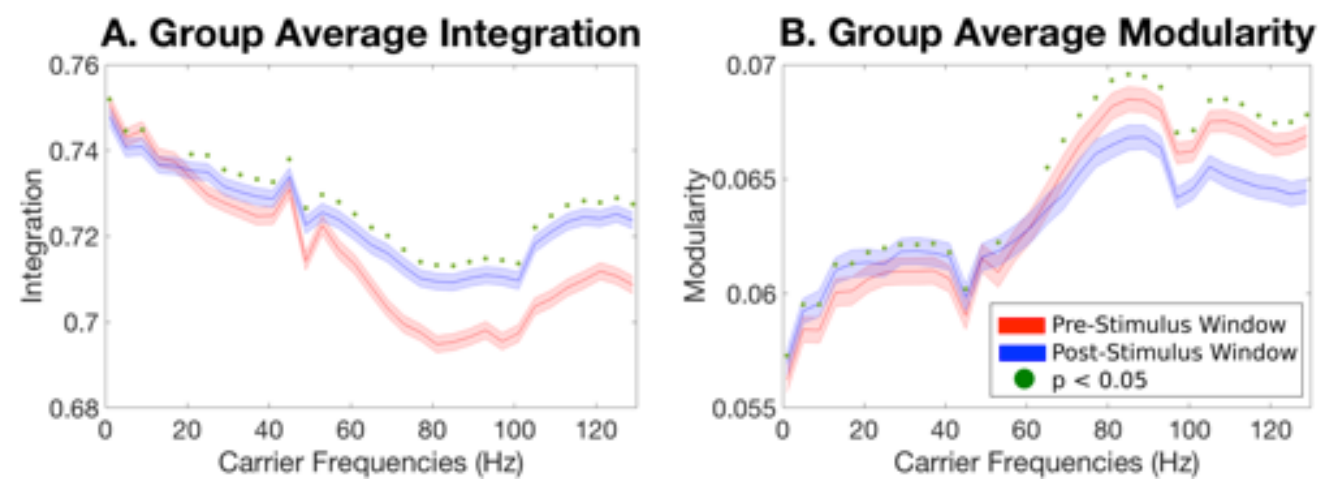

Figure 6. Group Analysis. Panel A shows a significant increase in integration during cognitive processing for almost all frequencies, but with the greatest effect in the gamma range (60 to $100 \mathrm{~Hz}$ ) as seen on each single subject. Complementary to the integration, panel B shows a decrease of the modularity which is more prominent in the high gamma range. The red line corresponds to pre-stimulus window, the blue line corresponds to poststimulus window, the shaded error regions reflect the standard deviation across trials and green dots indicates a statistical significance of $p<0.05(N=1000)$. 


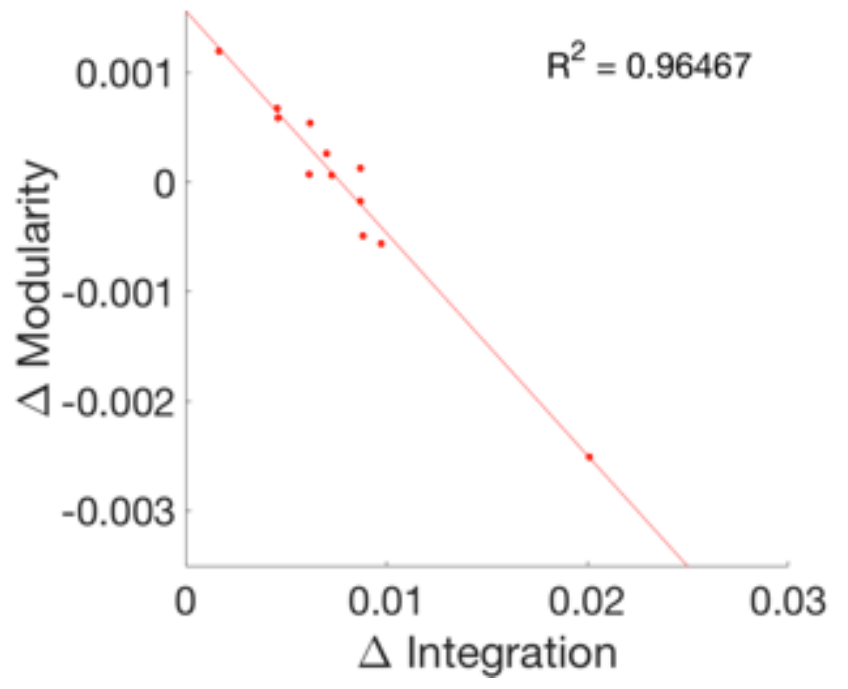

Figure 7. Correlation between Integration and Segregation. The figure shows the coefficient of determination between the integration and segregation measures across patients $(N=12)$. The $R^{2}$ represents the proportion of variance explained by a linear model. Note that both measures are highly correlated. 

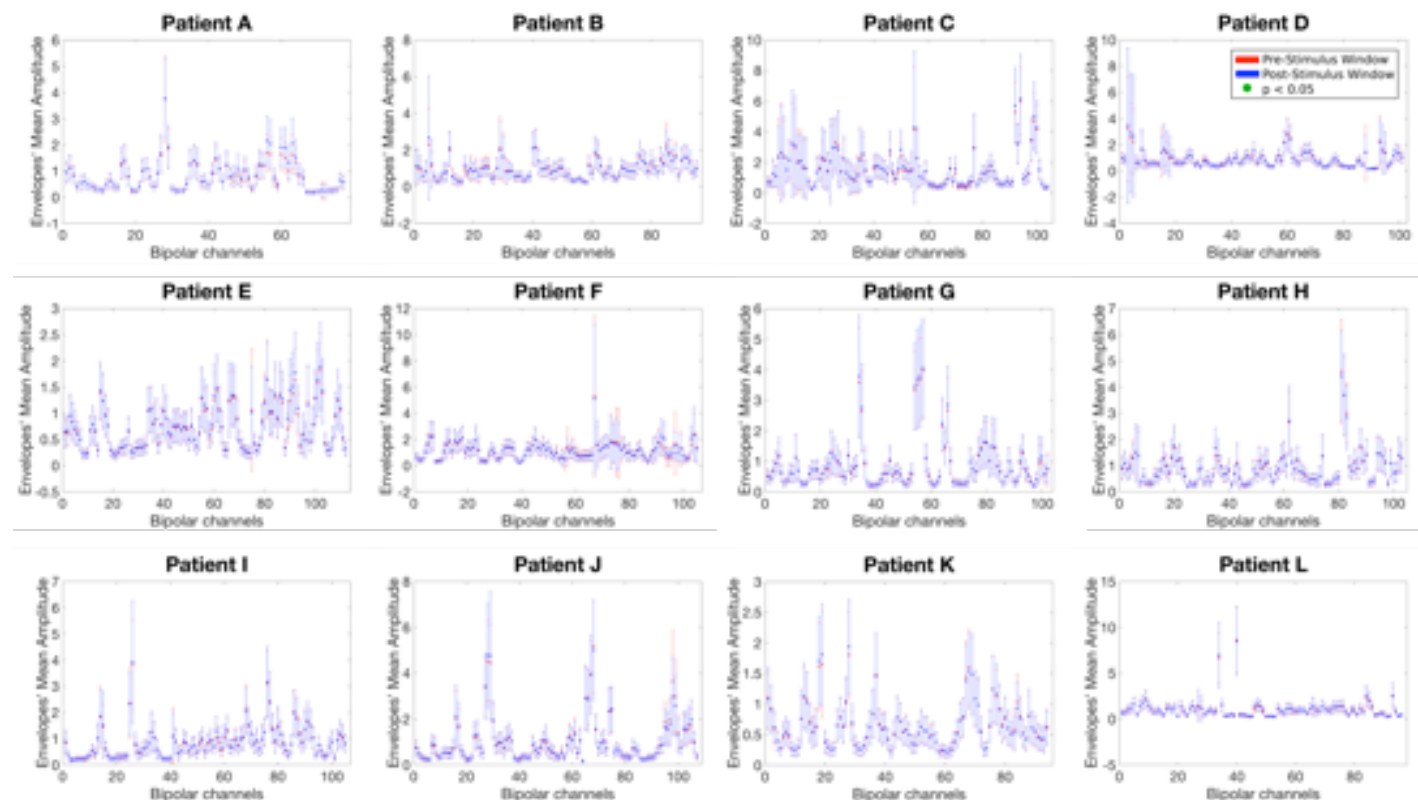

Figure 8. Envelopes' Amplitude at $60 \mathrm{~Hz}$ across electrodes for each patient. The panels show the results of the mean amplitude and SD at $60 \mathrm{~Hz}$ for each bipolar channel and both pre-and post-stimulus window for the picture-naming task. For all patients, there is no noticeable modulation across single bipolar channels between pre- and post-stimulus windows. The red line corresponds to pre-stimulus window and the blue line corresponds to post-stimulus window. The shaded error regions reflect the standard deviation across trials. Note the strong overlap of both lines. Green dots indicate a statistical significance of $p<0.05(N=1000)$. 
Lexical Decision Task
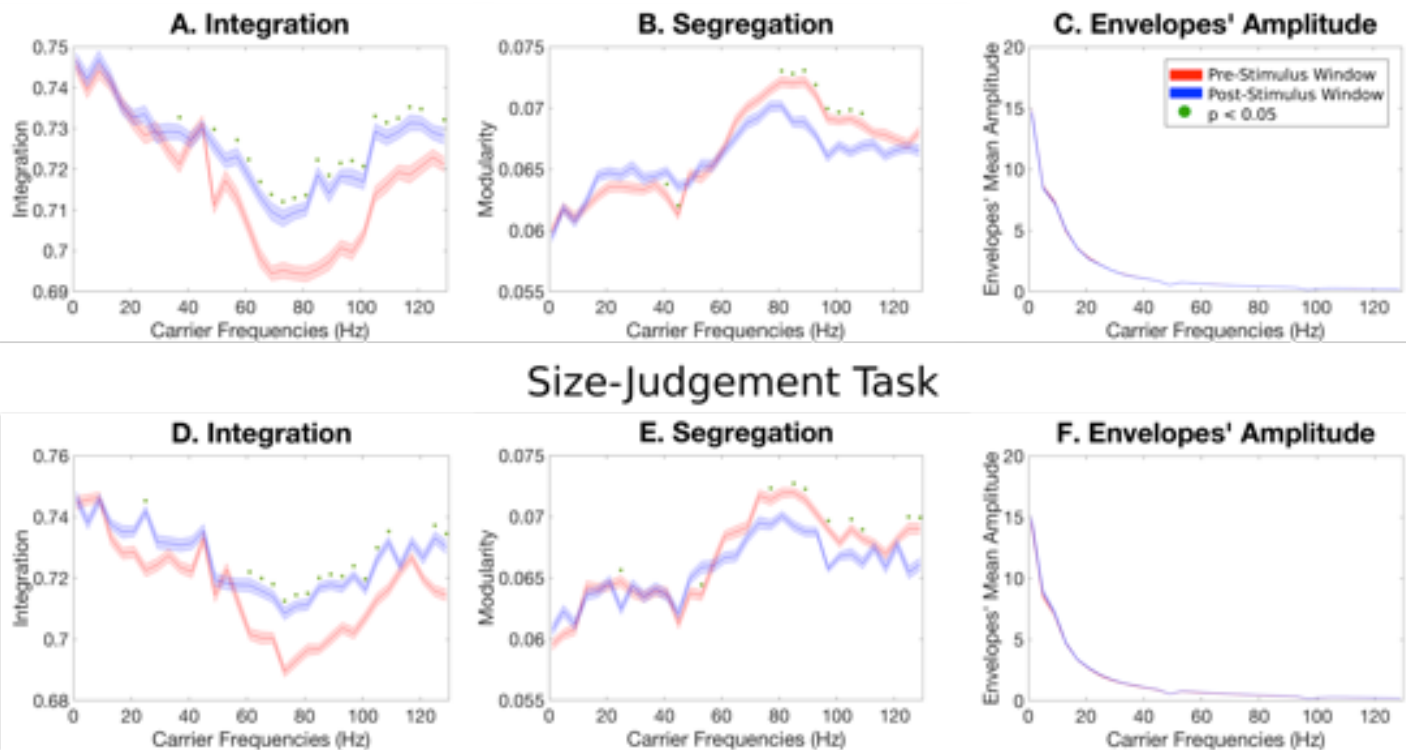

Figure 9. Results analysis patient K for the Lexical Decision and Size-Judgement Task.

Panels $A$ and $D$ shows a significant increase in the integration in the post-stimulus window (red line) compared to the post-stimulus window (blue line). The greatest effect is observed in the gamma range (50 to $90 \mathrm{~Hz}$ ). The shaded error regions reflect the standard deviation across trials. Green dots indicate a statistical significance of $p<0.05 \quad(N=1000)$. Complementary to the integration measure, panels $B$ and $E$ shows a decrease of Segregation in the same frequency range. Panels $C$ and $F$ shows that there are no oscillations' amplitude changes in any frequency induced by the stimulus (both curves are strongly overlapped). Note that the modulatory effect is almost the same for both tasks. 

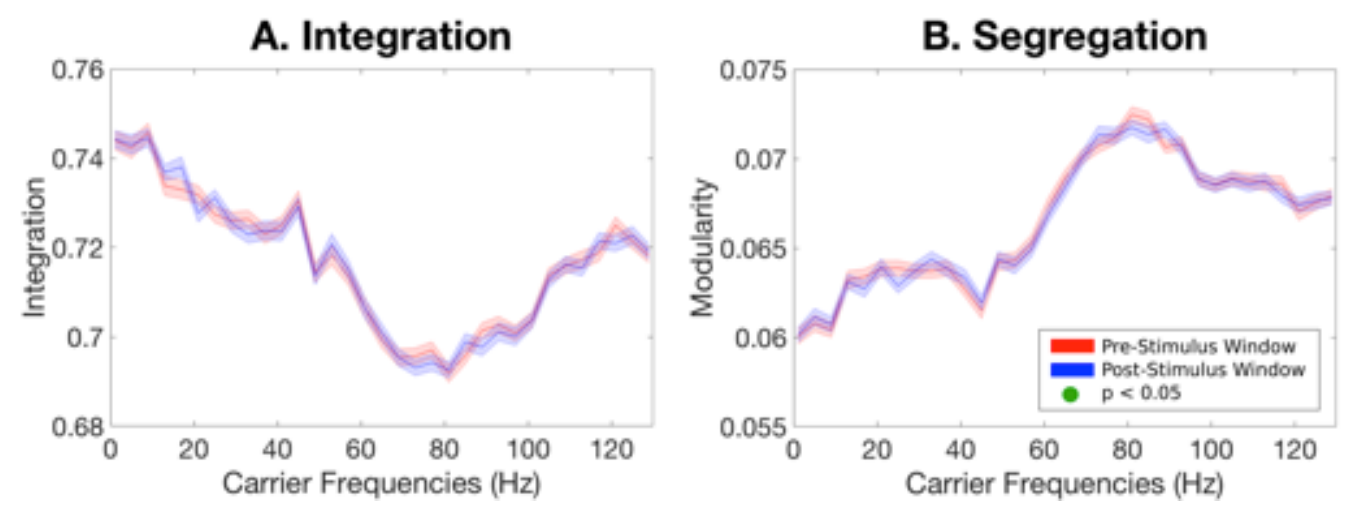

Figure 10. Inter pre-stimulus presentation window comparison for the picture-naming

task. Panel A shows the result of the measure of Integration, and panel B shows the result of the measure of Segregation, when contrasting half of pre-stimulus trials against the other half. There is no modulation of any of the two measures before stimulus presentation. The red line corresponds to 50\% of pre-stimulus windows; blue line corresponds to the other $50 \%$ of pre-stimulus window. The shaded error regions reflect the standard deviation across trials. Green dots indicate a statistical significance of $P<0.05(N=1000)$. 

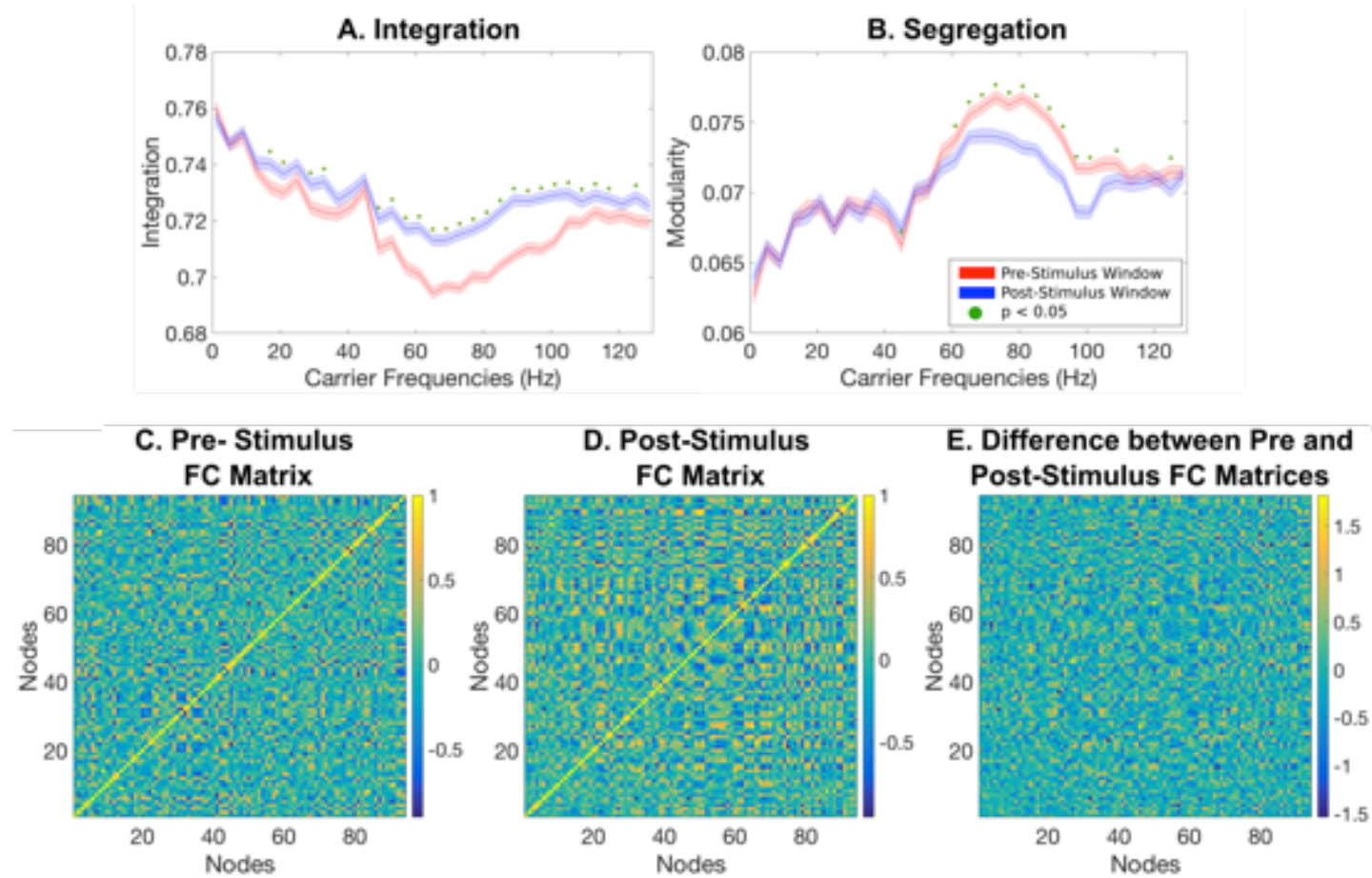

Figure 11. Results analysis using the FC matrix based on the monopolar montage for

Patient A. Panels $A$ and $B$ show the integration and segregation modulation during preand post-stimulus presentation, respectively, for the picture-naming task. The red line corresponds to pre-stimulus window, blue line corresponds to post-stimulus window. The shaded error regions reflect the standard deviation across trials and green dots indicate a statistical significance of $p<0.05(N=1000)$. Panels $C$ and $D$ shows the FC matrices computed for $60 \mathrm{~Hz}$ for the pre-and post-stimulus windows respectively. Panel E shows the difference between pre- and post-stimulus windows. The differences between both matrices (C and D, expressed on E), evidence the distributed character of the modulation across many different nodes. 

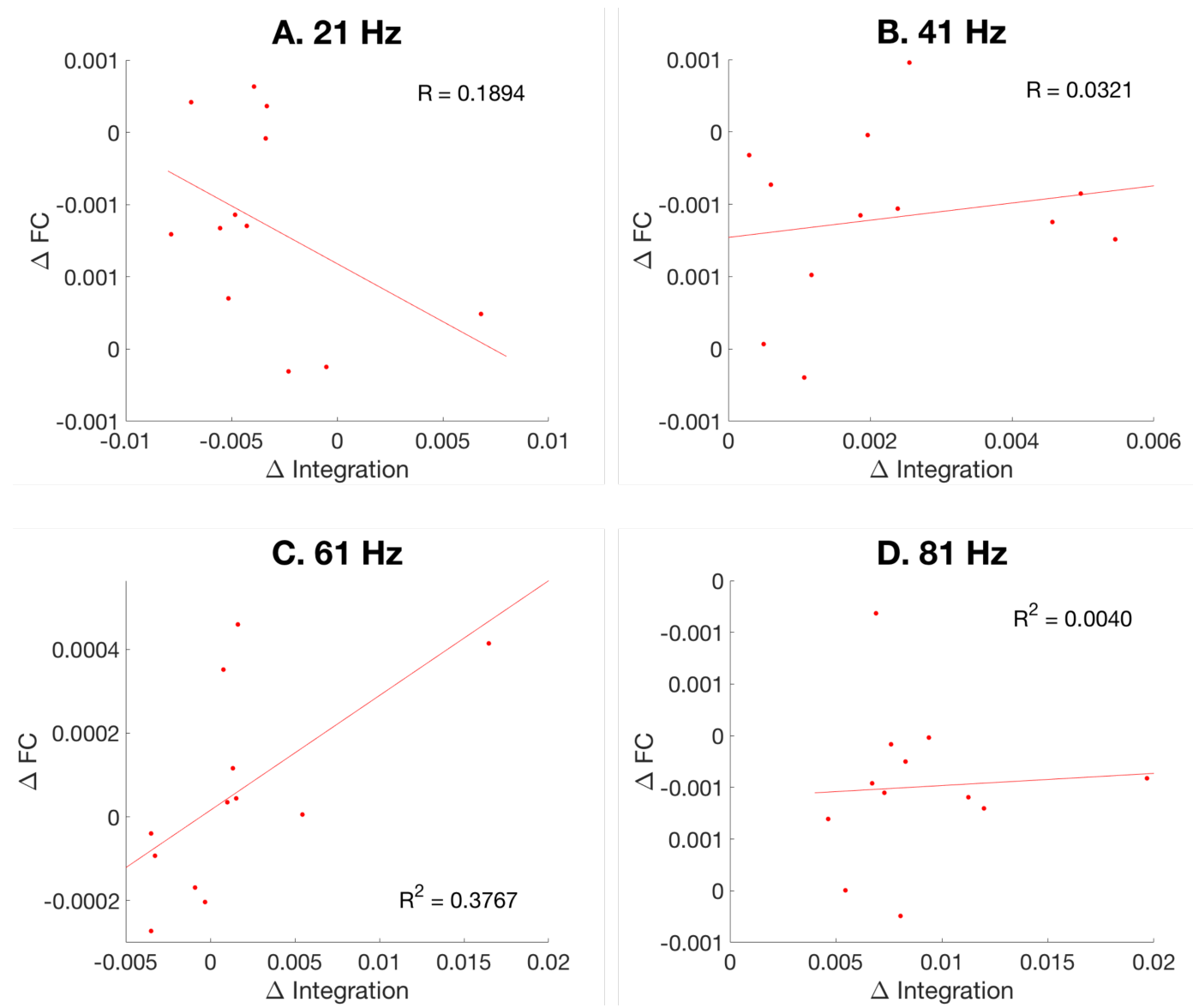

Figure 12. Correlation between Integration and Functional Connectivity. The figure shows the coefficient of determination between the integration and the mean variance of the functional connectivity calculated at $20,40,60$ and $80 \mathrm{~Hz}$ across the studied patients $(\mathrm{N}=12)$ based on the monopolar montage. The $\mathrm{R}^{2}$ represents the proportion of variance explained by a linear model. In all cases there is no correlation between both measures. 


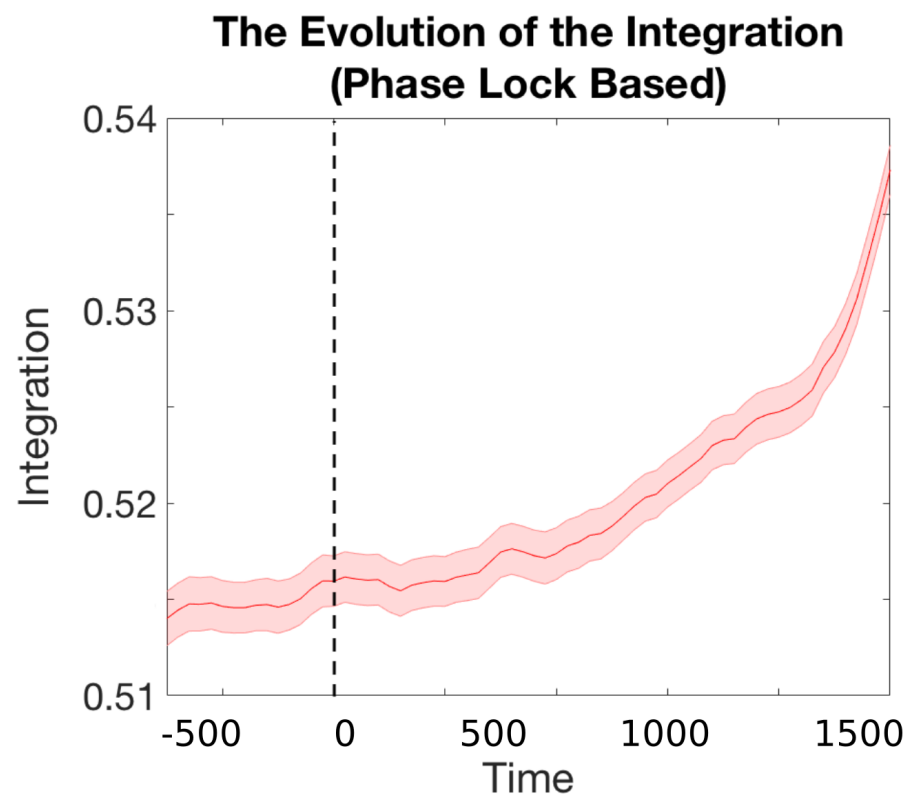

Figure 13. Time evolution of the Integration measure for Patient $\mathrm{K}$ during the picturenaming task. The figure shows the integration measure calculated for the phase-lock matrix for every single time point for the whole period of time at $80 \mathrm{~Hz}$. As seen, there is a monotonic increasing behaviour of the integration starting after the stimulus onset. The shaded error regions reflect the standard deviation across trials. 

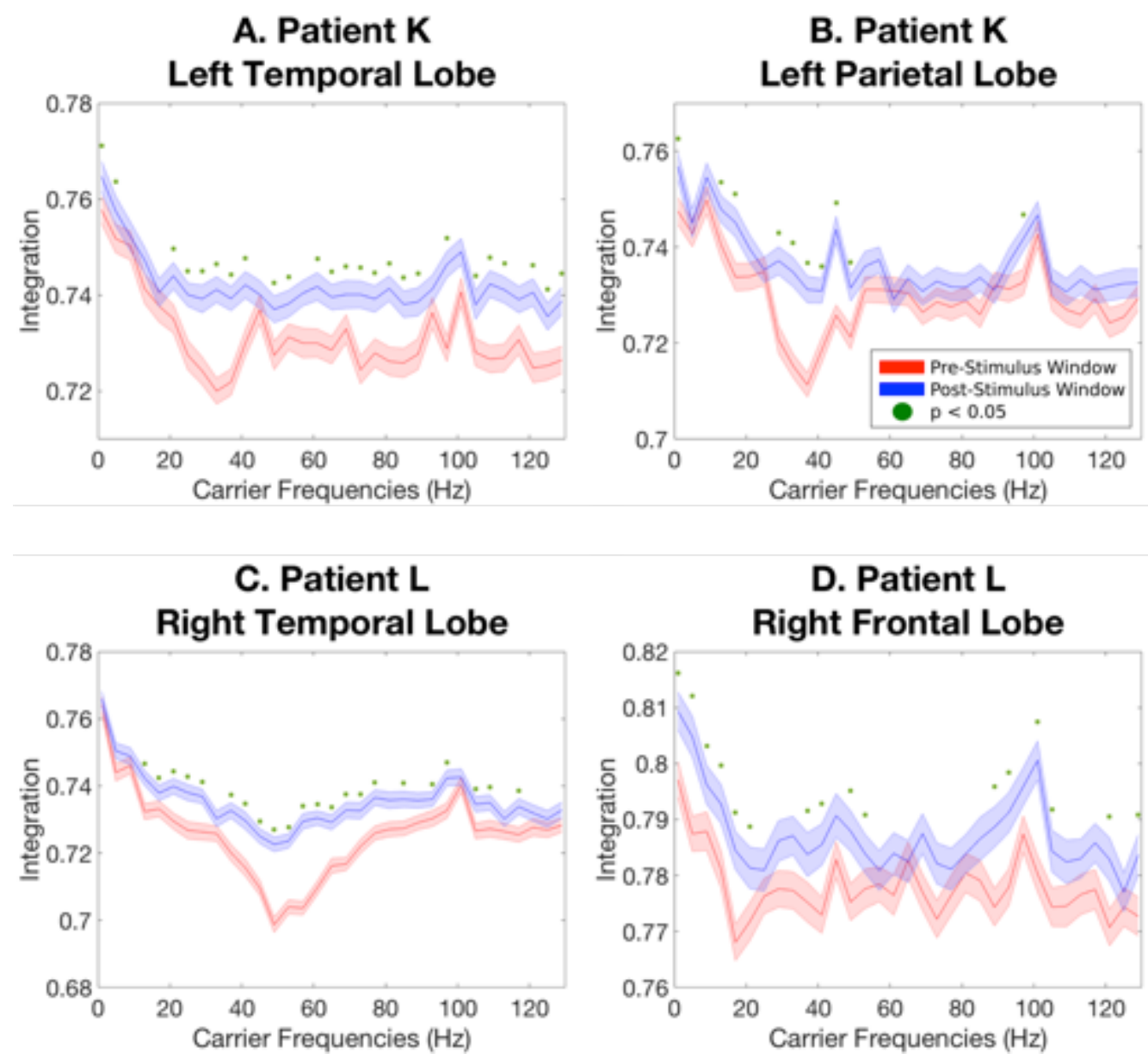

Figure 14. Results of the analysis of sub-networks within frontal, temporal and parietal lobes for the Picture-naming task. The upper panels show a significant increase of the integration in the post-stimulus window (red line) compared to the post-stimulus window (blue line) in the left Temporal (A) and left Parietal (B) lobes for patient K. The greatest effect is observed in the gamma range $(50$ to $90 \mathrm{~Hz})$. The shaded error regions reflect the standard deviation across trials. Green dots indicate a statistical significance of $p<0.05$ $(N=1000)$. Panels $(C)$ and $(D)$ shows the results of the right temporal and Frontal lobes respectively, for patient $L$. The modulatory effect is seen in the whole range of frequencies. 


\section{References}

1. Engel, A.K., et al., Invasive recordings from the human brain: clinical insights and beyond. Nat Rev Neurosci, 2005. 6(1): p. 35-47.

2. Lachaux, J.P., D. Rudrauf, and P. Kahane, Intracranial EEG and human brain mapping. J Physiol Paris, 2003. 97(4-6): p. 613-28.

3. Lachaux, J.P., et al., High-frequency neural activity and human cognition: past, present and possible future of intracranial EEG research. Prog Neurobiol, 2012. 98(3): p. 279-301.

4. Musch, K., et al., Selective attention modulates high-frequency activity in the faceprocessing network. Cortex, 2014. 60: p. 34-51.

5. Ossandon, T., et al., Efficient "pop-out" visual search elicits sustained broadband gamma activity in the dorsal attention network. J Neurosci, 2012. 32(10): p. 341421.

6. Bertrand, J.A., et al., Recognizing an object from the sum of its parts: an intracranial study on alpha rhythms. J Cogn Neurosci, 2014. 26(8): p. 1797-805.

7. Sahin, N.T., et al., Sequential processing of lexical, grammatical, and phonological information within Broca's area. Science, 2009. 326(5951): p. 445-9.

8. Chan, A.M., et al., First-pass selectivity for semantic categories in human anteroventral temporal lobe. J Neurosci, 2011. 31(49): p. 18119-29.

9. Hamame, C.M., et al., High frequency gamma activity in the left hippocampus predicts visual object naming performance. Brain Lang, 2014. 135: p. 104-14.

10. Kucewicz, M.T., et al., High frequency oscillations are associated with cognitive processing in human recognition memory. Brain, 2014. 137(Pt 8): p. 2231-44.

11. Greenberg, J.A., et al., Decreases in theta and increases in high frequency activity underlie associative memory encoding. Neuroimage, 2015. 114: p. 257-63.

12. Haque, R.U., et al., Cortical Low-Frequency Power and Progressive Phase Synchrony Precede Successful Memory Encoding. J Neurosci, 2015. 35(40): p. 13577-86.

13. Perez, O., et al., Preconscious prediction of a driver's decision using intracranial recordings. J Cogn Neurosci, 2015. 27(8): p. 1492-502.

14. Murray, R.J., T. Brosch, and D. Sander, The functional profile of the human amygdala in affective processing: insights from intracranial recordings. Cortex, 2014. 60: p. 10-33.

15. Boucher, O., et al., Spatiotemporal dynamics of affective picture processing revealed by intracranial high-gamma modulations. Hum Brain Mapp, 2015. 36(1): p. 16-28.

16. Gaillard, R., et al., Converging intracranial markers of conscious access. PLoS Biol, 2009. 7(3): p. e61. 
17. Kingyon, J., et al., High-gamma band fronto-temporal coherence as a measure of functional connectivity in speech motor control. Neuroscience, 2015. 305: p. 15-25.

18. Zheng, J., et al., Amygdala-hippocampal dynamics during salient information processing. Nat Commun, 2017. 8: p. 14413.

19. Mercier, M.R., et al., Neuro-oscillatory phase alignment drives speeded multisensory response times: an electro-corticographic investigation. J Neurosci, 2015. 35(22): p. 8546-57.

20. Kahana, M.J., The cognitive correlates of human brain oscillations. J Neurosci, 2006. 26(6): p. 1669-72.

21. Bressler, S.L. and V. Menon, Large-scale brain networks in cognition: emerging methods and principles. Trends Cogn Sci, 2010. 14(6): p. 277-90.

22. Deco, G., et al., Rethinking segregation and integration: contributions of wholebrain modelling. Nat Rev Neurosci, 2015. 16(7): p. 430-9.

23. De Vico Fallani, F., et al., Brain network analysis from high-resolution EEG recordings by the application of theoretical graph indexes. IEEE Trans Neural Syst Rehabil Eng, 2008. 16(5): p. 442-52.

24. Wang, N., L. Zhang, and G. Liu, EEG-based research on brain functional networks in cognition. Biomed Mater Eng, 2015. 26 Suppl 1: p. S1107-14.

25. Kitzbichler, M.G., et al., Cognitive effort drives workspace configuration of human brain functional networks. J Neurosci, 2011. 31(22): p. 8259-70.

26. Palva, S., S. Monto, and J.M. Palva, Graph properties of synchronized cortical networks during visual working memory maintenance. Neuroimage, 2010. 49(4): $p$. 3257-68.

27. Valencia, M., et al., Dynamic small-world behavior in functional brain networks unveiled by an event-related networks approach. Phys Rev E Stat Nonlin Soft Matter Phys, 2008. 77(5 Pt 1): p. 050905.

28. Bassett, D.S., et al., Dynamic reconfiguration of human brain networks during learning. Proc Natl Acad Sci U S A, 2011. 108(18): p. 7641-6.

29. Chai, L.R., et al., Functional Network Dynamics of the Language System. Cereb Cortex, 2016.

30. Ekman, M., et al., Predicting errors from reconfiguration patterns in human brain networks. Proc Natl Acad Sci U S A, 2012. 109(41): p. 16714-9.

31. Kinnison, J., et al., Network analysis reveals increased integration during emotional and motivational processing. J Neurosci, 2012. 32(24): p. 8361-72.

32. Wang, X., et al., The Hierarchical Structure of the Face Network Revealed by Its Functional Connectivity Pattern. J Neurosci, 2016. 36(3): p. 890-900.

33. Bola, M. and B.A. Sabel, Dynamic reorganization of brain functional networks during cognition. Neuroimage, 2015. 114: p. 398-413.

34. Vatansever, D., et al., Default Mode Dynamics for Global Functional Integration. J Neurosci, 2015. 35(46): p. 15254-62. 
35. Godwin, D., R.L. Barry, and R. Marois, Breakdown of the brain's functional network modularity with awareness. Proc Natl Acad Sci U S A, 2015. 112(12): $p$. 3799-804.

36. Liang, X., et al., Topologically Reorganized Connectivity Architecture of DefaultMode, Executive-Control, and Salience Networks across Working Memory Task Loads. Cereb Cortex, 2016. 26(4): p. 1501-11.

37. Becher, A.K., et al., Intracranial electroencephalography power and phase synchronization changes during monaural and binaural beat stimulation. European Journal of Neuroscience, 2015. 41(2): p. 254-263.

38. Axmacher, N., et al., Interactions between medial temporal lobe, prefrontal cortex, and inferior temporal regions during visual working memory: a combined intracranial EEG and functional magnetic resonance imaging study. Journal of Neuroscience, 2008. 28(29): p. 7304-7312.

39. Deco, G. and M.L. Kringelbach, Metastability and Coherence: Extending the Communication through Coherence Hypothesis Using A Whole-Brain Computational Perspective. Trends Neurosci, 2016. 39(3): p. 125-35.

40. Fries, P., A mechanism for cognitive dynamics: neuronal communication through neuronal coherence. Trends Cogn Sci, 2005. 9(10): p. 474-80.

41. Fries, P., Neuronal gamma-band synchronization as a fundamental process in cortical computation. Annu Rev Neurosci, 2009. 32: p. 209-24.

42. Fries, P., Rhythms for Cognition: Communication through Coherence. Neuron, 2015. 88(1): p. 220-35.

43. Womelsdorf, T., et al., Gamma-band synchronization in visual cortex predicts speed of change detection. Nature, 2006. 439(7077): p. 733-6.

44. Howard, M.W., et al., Gamma oscillations correlate with working memory load in humans. Cereb Cortex, 2003. 13(12): p. 1369-74.

45. Ball, T., et al., Movement related activity in the high gamma range of the human EEG. Neuroimage, 2008. 41(2): p. 302-10.

46. Jones, M.W. and M.A. Wilson, Theta rhythms coordinate hippocampal-prefrontal interactions in a spatial memory task. PLoS Biol, 2005. 3(12): p. e402.

47. Fell, J., et al., Human memory formation is accompanied by rhinal-hippocampal coupling and decoupling. Nat Neurosci, 2001. 4(12): p. 1259-64.

48. Gregoriou, G.G., et al., High-frequency, long-range coupling between prefrontal and visual cortex during attention. Science, 2009. 324(5931): p. 1207-10.

49. Roelfsema, P.R., et al., Visuomotor integration is associated with zero time-lag synchronization among cortical areas. Nature, 1997. 385(6612): p. 157-61.

50. Jerbi, K., et al., Task-related gamma-band dynamics from an intracerebral perspective: review and implications for surface EEG and MEG. Hum Brain Mapp, 2009. 30(6): p. 1758-71.

51. Burke, J.F., et al., Synchronous and asynchronous theta and gamma activity during episodic memory formation. J Neurosci, 2013. 33(1): p. 292-304. 
52. Brookes, M.J., et al., Investigating the electrophysiological basis of resting state networks using magnetoencephalography. Proc Natl Acad Sci U S A, 2011. 108(40): p. 16783-8.

53. Brookes, M.J., et al., Measuring functional connectivity using MEG: methodology and comparison with fcMRI. Neuroimage, 2011. 56(3): p. 1082-104.

54. Cabral, J., et al., Exploring mechanisms of spontaneous functional connectivity in MEG: how delayed network interactions lead to structured amplitude envelopes of band-pass filtered oscillations. Neuroimage, 2014. 90: p. 423-35.

55. Nir, Y., et al., Interhemispheric correlations of slow spontaneous neuronal fluctuations revealed in human sensory cortex. Nat Neurosci, 2008. 11(9): p. 11008.

56. Rubinov, M. and O. Sporns, Weight-conserving characterization of complex functional brain networks. Neuroimage, 2011. 56(4): p. 2068-79.

57. Newman, M.E., Modularity and community structure in networks. Proc Natl Acad Sci U S A, 2006. 103(23): p. 8577-82.

58. Sporns, O., Networks of the Brain. 2010: The MIT Press. 423.

59. Sperling, M.R., Clinical challenges in invasive monitoring in epilepsy surgery. Epilepsia, 1997. 38 Suppl 4: p. S6-12.

60. Serletis, D., et al., The stereotactic approach for mapping epileptic networks: a prospective study of 200 patients. J Neurosurg, 2014. 121(5): p. 1239-46.

61. Ferstl, E.C., et al., The extended language network: a meta-analysis of neuroimaging studies on text comprehension. Hum Brain Mapp, 2008. 29(5): $p$. 581-93.

62. Price, C.J., The anatomy of language: contributions from functional neuroimaging. J Anat, 2000. 197 Pt 3: p. 335-59.

63. Winkler, A.M., et al., Permutation inference for the general linear model. Neuroimage, 2014. 92: p. 381-397.

64. Tononi, G., O. Sporns, and G.M. Edelman, A measure for brain complexity: relating functional segregation and integration in the nervous system. Proc Natl Acad Sci U S A, 1994. 91(11): p. 5033-7.

65. Fox, P.T. and K.J. Friston, Distributed processing; distributed functions? Neuroimage, 2012. 61(2): p. 407-26.

66. Sporns, O., Network attributes for segregation and integration in the human brain. Curr Opin Neurobiol, 2013. 23(2): p. 162-71.

67. Power, J.D., et al., Functional network organization of the human brain. Neuron, 2011. 72(4): p. 665-678.

68. Tononi, G., G.M. Edelman, and O. Sporns, Complexity and coherency: integrating information in the brain. Trends in cognitive sciences, 1998. 2(12): p. 474-484.

69. Tononi, G. and O. Sporns, Measuring information integration. BMC neuroscience, 2003. 4(1): p. 31. 
70. Barrett, A.B. and A.K. Seth, Practical measures of integrated information for timeseries data. PLoS computational biology, 2011. 7(1): p. e1001052.

71. Oizumi, M., L. Albantakis, and G. Tononi, From the phenomenology to the mechanisms of consciousness: integrated information theory 3.0. PLoS computational biology, 2014. 10(5): p. e1003588.

72. Elton, A. and W. Gao, Task-related modulation of functional connectivity variability and its behavioral correlations. Hum Brain Mapp, 2015. 36(8): p. 3260-72.

73. Sporns, O. and R.F. Betzel, Modular Brain Networks. Annu Rev Psychol, 2016. 67: p. $613-40$.

The dynamics of human cognition: increasing global integration coupled with decreasing segregation found using iEEG

Josephine Cruzat*a, Gustavo Deco ${ }^{\mathrm{a}, \mathrm{b}, \mathrm{c}, \mathrm{d}}$, Adrià Tauste Campo ${ }^{\mathrm{a}, \mathrm{e}}$, Alessandro Principe ${ }^{\mathrm{e}}$,

Albert Costa $^{\mathrm{a}, \mathrm{b}}$, Morten L. Kringelbach ${ }^{\mathrm{f}, \mathrm{g}, \mathrm{h}}$ \& Rodrigo Rocamora ${ }^{\mathrm{e}}$

\section{Supplementary Material}



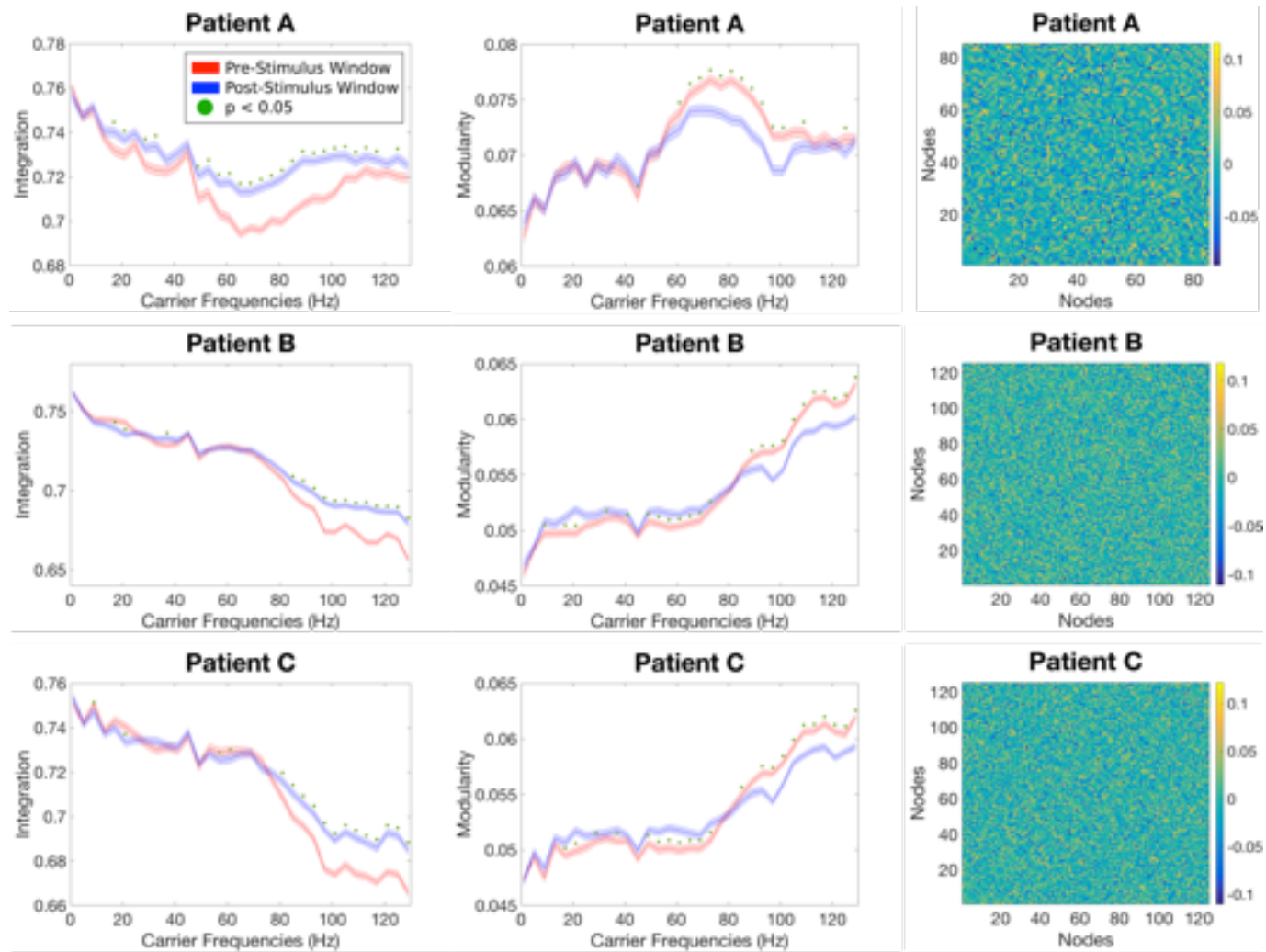

Patient C
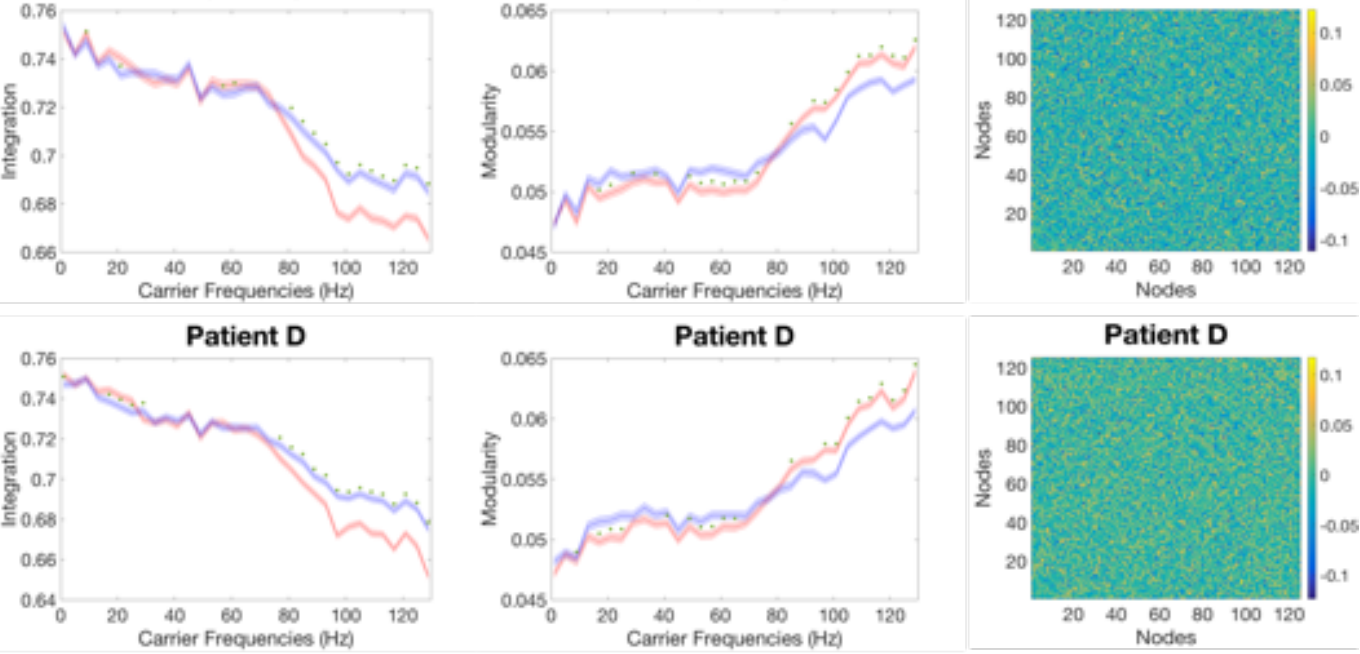

Patient D

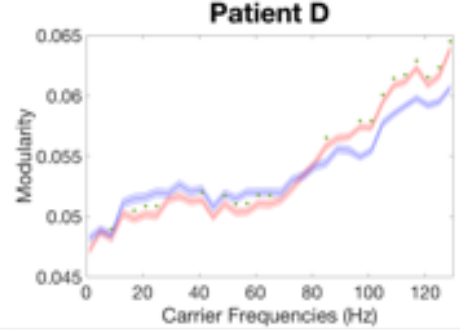

Patient D
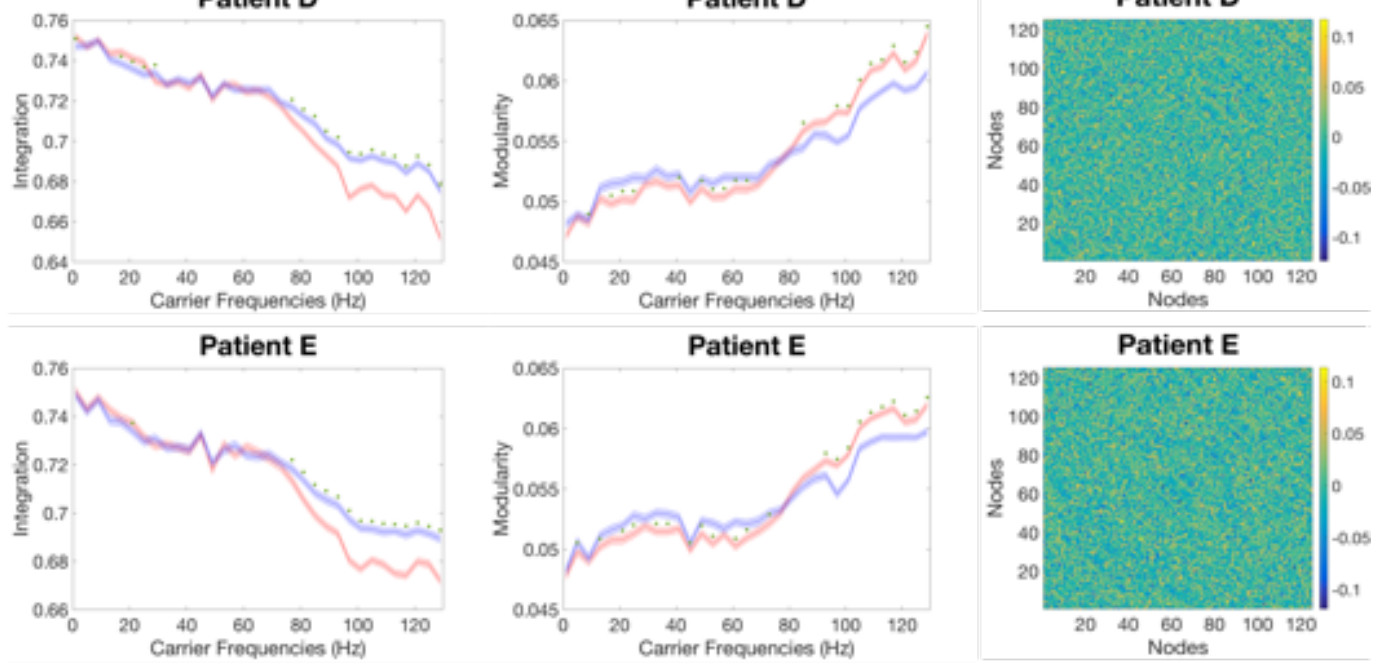

Patient E

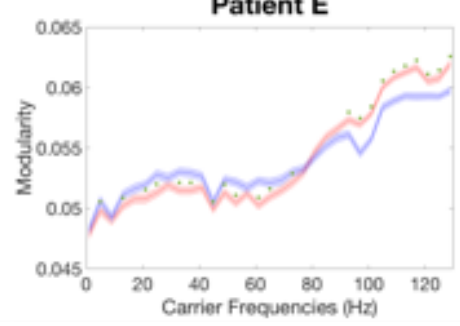

Patient E
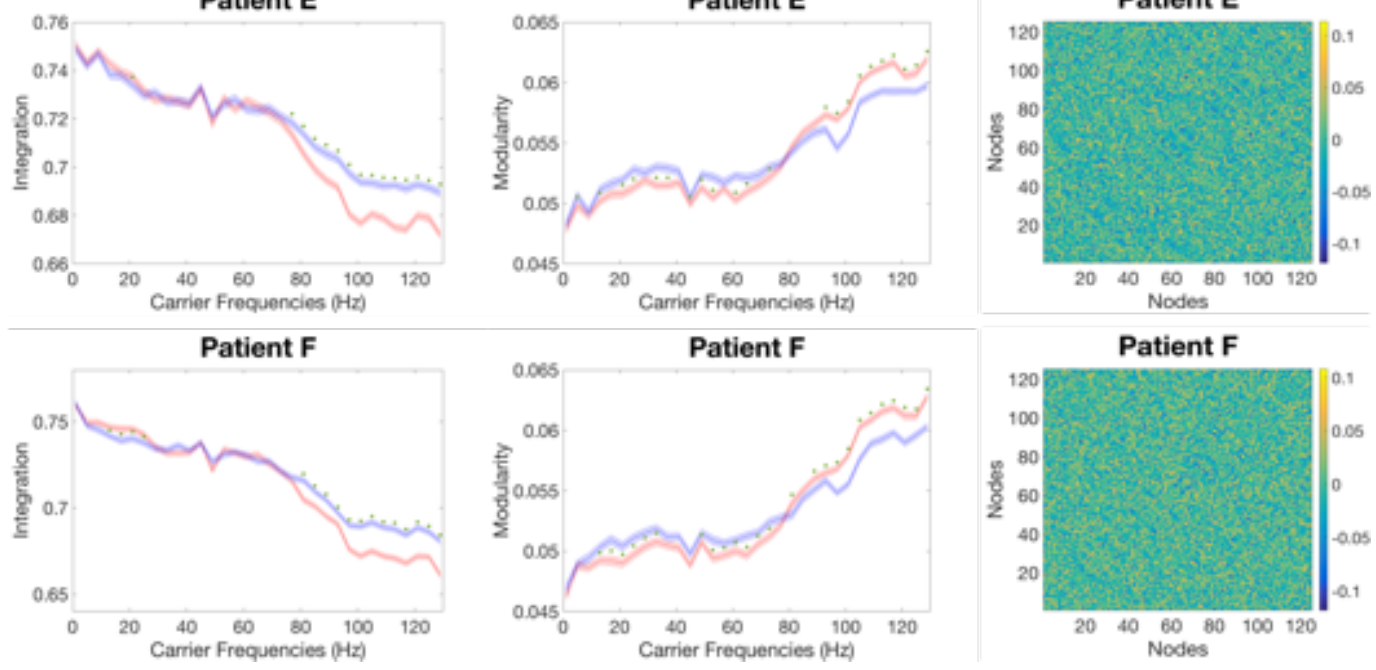

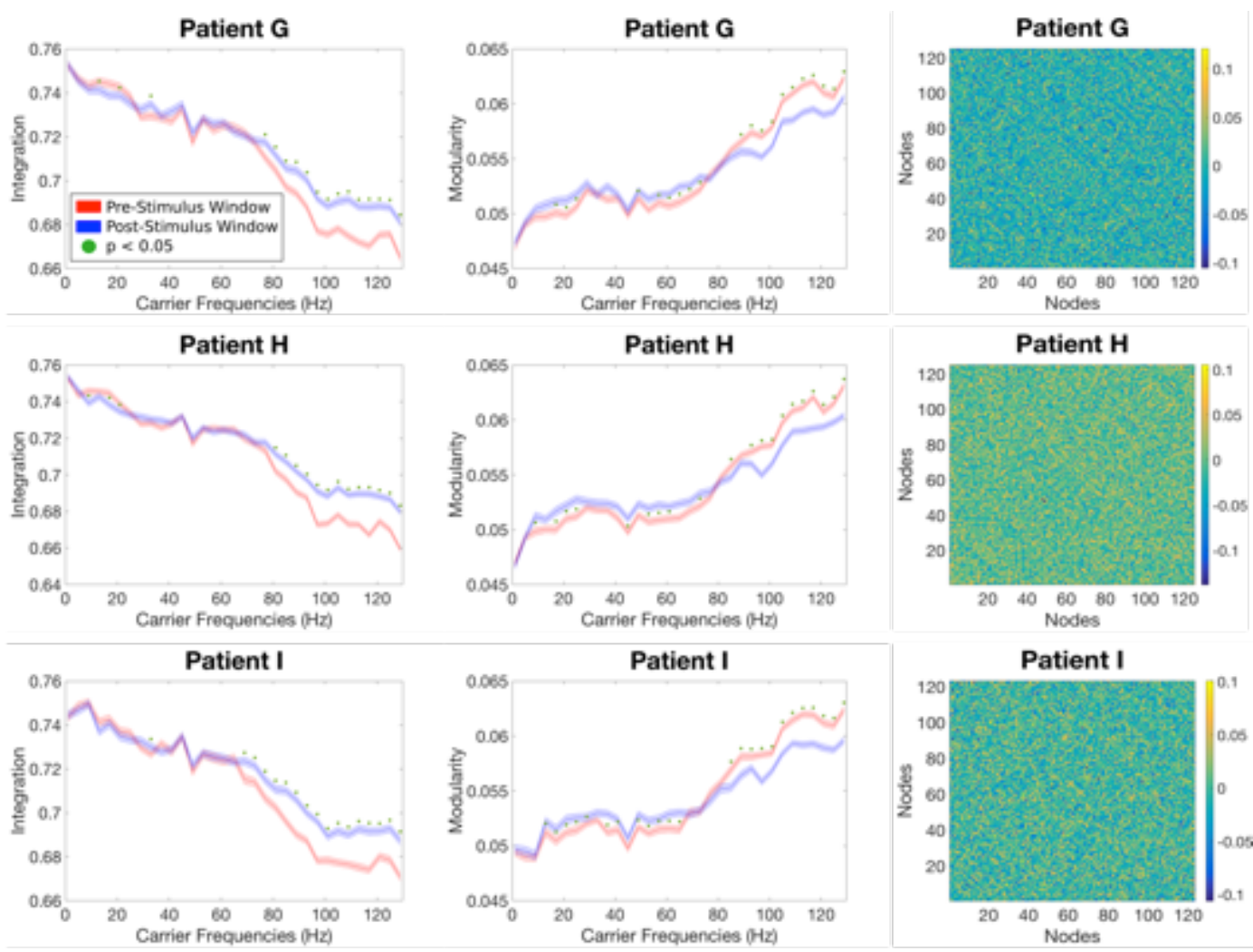

Patient I

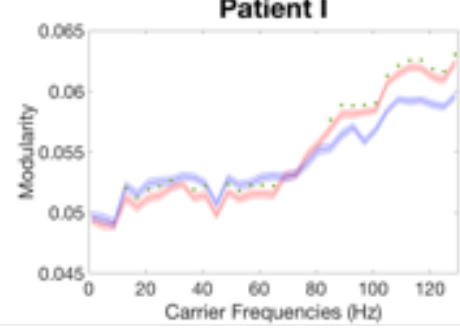

Patient I

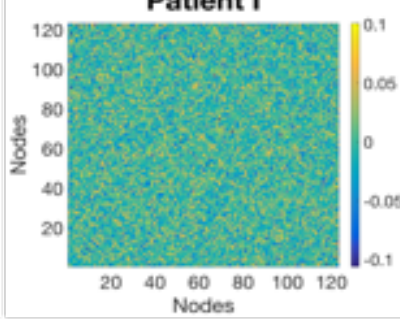

Patient

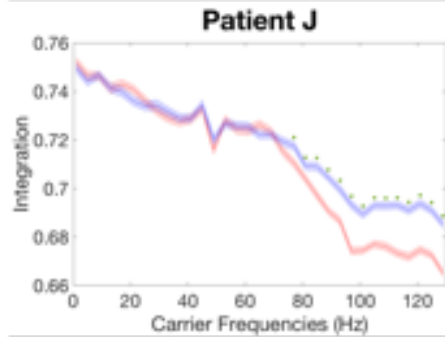

Patient J
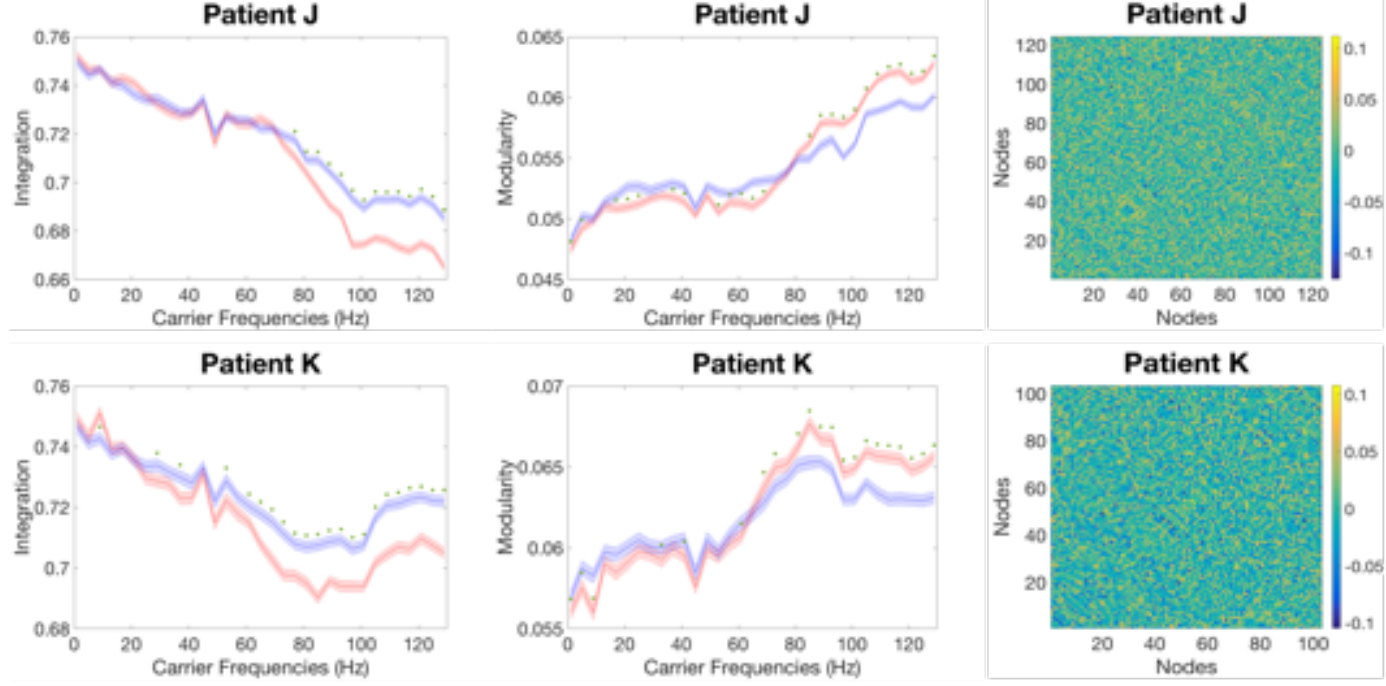

Patient L

Patient L
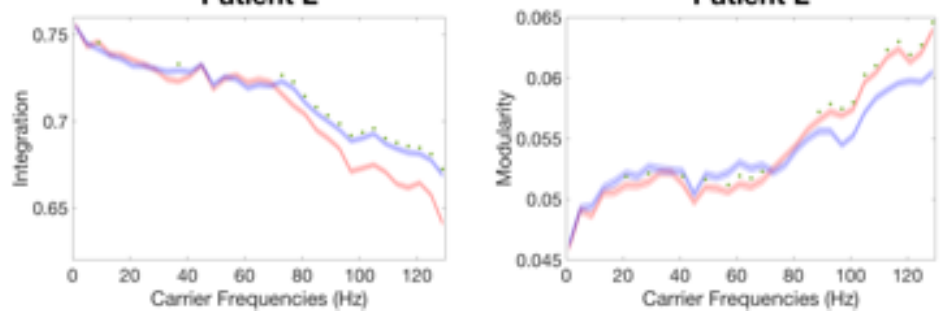

Patient L

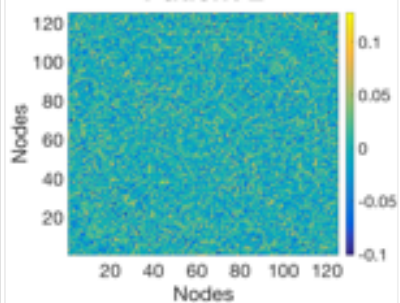


Figure S1. Integration measure using the FC matrices based on the monopolar montage for each participant. The panels in the left column show the integration modulation during pre- and post-stimulus presentation, respectively, for the picture-naming task. The modulation is seen in the whole range of frequencies. Panels in the middle column show the modulation of the segregation (measured by the modularity) during pre-and poststimulus presentation, respectively, for the same task. Even though the difference of both windows is significant, note that the modulation is much smaller than the obtained when using the bipolar montage. The red line corresponds to pre-stimulus window, blue line corresponds to post-stimulus window. The shaded error regions reflect the standard deviation across trials and green dots indicates a statistical significance of $p<0.05$ $(N=1000)$. The panels in the right column show the difference between pre-and poststimulus windows FC matrices, based on the monopolar montage and computed for $60 \mathrm{~Hz}$. The panels evidence the distributed character of the modulation across many different nodes. 